FEDERAL RESERVE BANK OF SAN FRANCISCO

WORKING PAPER SERIES

\title{
On the Inefficiency of Non-Competes in Low-Wage Labor Markets
}

\author{
Tristan Potter \\ Drexel University \\ Bart Hobijn \\ Arizona State University \\ Federal Reserve Bank of San Francisco \\ Andre Kurmann \\ Drexel University \\ January 2022 \\ Working Paper 2022-01
}

https://www.frbsf.org/economic-research/publications/working-papers/2022/01/

\section{Suggested citation:}

Potter, Tristan, Bart Hobijn, Andre Kurmann. 2022 "On the Inefficiency of NonCompetes in Low-Wage Labor Markets," Federal Reserve Bank of San Francisco Working Paper 2022-01. https://doi.org/10.24148/wp2022-01

The views in this paper are solely the responsibility of the authors and should not be interpreted as reflecting the views of the Federal Reserve Bank of San Francisco or the Board of Governors of the Federal Reserve System. 


\title{
On the Inefficiency of Non-Competes in Low-Wage Labor Markets*
}

\author{
Tristan Potter \\ Drexel University
}

\author{
Bart Hobijn \\ Arizona State University \\ Federal Reserve Bank of San Francisco
}

\author{
André Kurmann \\ Drexel University
}

January 18,2022

\begin{abstract}
We study the efficiency of non-compete agreements (NCAs) in an equilibrium model of labor turnover. The model is consistent with empirical studies showing that NCAs reduce turnover, average wages, and wage dispersion for low-wage workers. But the model also predicts that NCAs, by reducing turnover, raise recruitment and employment. We show that optimal NCA policy: (i) is characterized by a Hosios-like condition that balances the benefits of higher employment against the costs of inefficient congestion and poaching; (ii) depends critically on the minimum wage, such that enforcing NCAs can be efficient with a sufficiently high minimum wage; and (iii) alone cannot always achieve efficiency, also true of a minimum wage - yet with both instruments efficiency is always attainable. To guide policy makers, we derive a sufficient statistic in the form of an easily computed employment threshold above which NCAs are necessarily inefficiently restrictive, and show that employment levels in current low-wage U.S. labor markets are typically above this threshold. Finally, we calibrate the model to show that Oregon's 2008 ban of NCAs for low-wage workers increased welfare, albeit modestly (by roughly $0.1 \%$ ), and that if policy makers had also raised the minimum wage to its optimal level (a 30\% increase), welfare would have increased more substantially-by over $1 \%$.
\end{abstract}

Keywords: Non-compete agreements; low-wage labor markets; minimum wage

JEL Classification: J62, J63, E24

${ }^{*}$ Thanks to Alan Manning for helpful comments and suggestions. The views expressed in this paper reflect those of the authors and not necessarily those of the Federal Reserve Bank of San Francisco or the Federal Reserve System. 
"In recent years, the wide use of non-competes has spread from highly technical fields into less technical and lower wage work, where they might reduce wage and benefit competition among employers and restrict employees' upward mobility...We would like the GAO to assess what is known about the effects of non-compete agreements on the workforce and the economy, including employment, wages and benefits"

- Letter from the U.S. Senate to the GAO (March 7, 2019) ${ }^{1}$

\section{Introduction}

Non-compete agreements (NCAs) - and post-employment restrictive covenants more broadly — have become a pervasive feature of low-wage labor contracts, including among several high-profile lowwage employers such as Amazon, Jimmy John's, McDonald's, Burger King, and Jiffy Lube (for example, see Krueger and Ashenfelter (2018), Colvin and Shierholz (2019), or Starr et al. (2021)). This has led to calls to prohibit the use of NCAs in low-wage labor markets, on the grounds that they unnecessarily prevent low-skilled workers from seeking better employment opportunities and higher wages. ${ }^{2}$

Absent from this debate, however, is an equilibrium analysis of the implications of NCAs for efficiency in low-wage labor markets - an analysis that is necessary to answer a number of important questions about NCA policy: Do NCAs impose negative externalities on the economy when the usual rationales for their use are not applicable? If so, should NCAs be completely banned for low-wage workers, as in Oregon in 2008 and a number of other states more recently? ${ }^{3}$ How do NCAs interact with minimum wages, another policy available to raise wages in low-wage labor markets? What is the welfare-maximizing level of NCA enforcement, and are there combinations of NCA and minimum wage policies that achieve efficiency?

The present paper provides answers to these questions. We consider a general equilibrium on-the-job search model with wage posting and endogenous job creation in the spirit of Burdett and Mortensen (1998) as extended by Manning (2003), and generalize it to accommodate NCAs that impede job-to-job transitions. Our focus on wage posting rather than bargaining reflects evidence that bargaining over wages is uncommon in low-wage sectors of the economy. ${ }^{4}$ Our focus on firms' recruitment decisions and thus job creation as the main mechanism through which NCAs impact efficiency reflects the view that other mechanisms commonly thought to bear on the efficiency of NCAs, such as protecting firms' investments in general human capital or intellectual property

\footnotetext{
${ }^{1}$ The quotation is an excerpt from a 2019 letter from senators Murphy (D-Ct), Young (R-In), Warren (D-Ma), Rubio (R-Fl), Kaine (D-Va) and Wyden (D-Or) to the Government Accountability Office (GAO).

${ }^{2}$ See the epigraph for expressed concern among policy makers. For examples of media coverage, see The New York Times: "How Non-Compete Clauses Keep Workers Locked In"; The Washington Post: "Even janitors have noncompetes now. Nobody is safe."; Financial Times: "Cushman v the cleaner: The fight over non-competes"; and The Wall Street Journal: "The Noncompete Agreement is Now Ridiculously Abused".

${ }^{3}$ Since 2017, eight additional states have restricted the use of NCAs for low-wage workers: Illinois (2017), Massachusetts (2018), New Hampshire (2019), Maine (2019), Maryland (2019), Washington (2020), Rhode Island (2020) and Virginia (2020).

${ }^{4}$ For example, Hall and Krueger (2012) find that less than one third of workers without a high school degree report bargaining over wages. For additional evidence, see Brenzel et al. (2014) and Doniger and Toohey (2021).
} 
considerations, are relatively unimportant for low-wage labor markets. ${ }^{5}$ We do not incorporate other margins through which NCAs could conceivably affect welfare in low-wage labor markets, such as participation decisions, preferring instead to focus our attention on job creation, which we view as the natural starting place for an equilibrium analysis of NCAs in low-wage labor markets. As we will see, our focus on job creation by itself yields a rich set of insights into the positive and normative equilibrium implications of NCAs. ${ }^{6}$

The analysis proceeds in three parts. In the first part, we show that, consistent with recent empirical evidence by Lipsitz and Starr (2021) on Oregon's NCA ban for low-wage workers, the model implies that NCAs have a negative effect on job-to-job transitions, average wages, and wage dispersion. But the model also predicts that NCAs stimulate job creation via recruitment and thus increase employment. This result, which is central for the ensuing welfare analysis, is not obvious ex ante: On the one hand, NCAs make poaching more difficult and thus reduce firms' incentives to engage in costly recruiting. On the other hand, NCAs increase the expected duration of a job match, thereby increasing firms' incentives to recruit. Our analysis establishes that the latter effect unambiguously dominates the former.

The second part of the analysis considers the (in)efficiency of NCAs. While NCAs result in more recruitment and higher employment, they also exacerbate negative externalities from individual firms' recruitment decisions - congestion and unproductive poaching. ${ }^{7}$ Thus, the decentralized equilibrium can feature either excessive or insufficient aggregate recruitment. Efficiency requires balancing these forces. We show that efficiency obtains in equilibrium if and only if a condition analogous to the well-known Hosios condition (Hosios, 1990) holds. This condition equates the social benefits of more recruitment effort - job creation and hence higher employment — with the social costs - unproductive turnover between equally productive firms and congestion externalities.

The efficiency condition depends on both the extent of NCA enforcement as well as the level of the minimum wage. This means that it is important to jointly consider the two policy instruments for the implementation of labor market efficiency rather than focusing on each policy separately. In fact, we show that neither optimal NCA policy nor optimal minimum wage policy on its own is sufficient to guarantee efficiency. However, efficiency can always be achieved with an appropriate combination of the two policies. The basic insight behind this result is that the two policy tools are constrained in opposite directions with respect to their ability to influence recruitment and thus efficiency, so while alone neither policy can guarantee efficiency, with both instruments efficiency can always be achieved.

Our theoretical characterization of efficiency produces novel insights into the channels through which NCAs influence social welfare. By itself, however, this offers little guidance for policy makers seeking to decide on the optimal use of NCAs in low-wage labor markets. The third part of the analysis provides such guidance. We start by deriving a sufficient statistic in the form of a simple employment

\footnotetext{
${ }^{5}$ We note that recruitment costs in our model can be interpreted as firm-specific training costs, which seem more likely to be relevant in low-wage labor markets.

${ }^{6}$ In Appendix E, we show that all of our qualitative results remain intact in a model with free entry instead of endogenous recruitment.

${ }^{7}$ See Coles and Mortensen (2016) for a discussion of unproductive poaching in a different context.
} 
threshold above which NCAs are necessarily too restrictive. This threshold only depends on the elasticity of the match function and, if the minimum wage is binding, the ratio of the labor share of income to the mean-min wage ratio - moments that are all readily available from the data. For empirically relevant values of these parameters based on data from low-wage labor markets, such as the Food Services and Drinking Places industry (NAICS 722) frequently analyzed in studies of the minimum wage, we find that NCAs are likely to be excessively restrictive and should therefore be weakened on efficiency grounds.

While the sufficient statistic analysis provides guidance on whether NCAs should be weakened, it does not provide a specific optimal policy recommendation, nor does it allow us to quantify the welfare implications of banning NCAs. To provide such a quantitative assessment, we calibrate the full model to low-wage labor markets in Oregon prior to its 2008 NCA ban and study the implications of prohibiting the use of NCAs in low-wage labor markets. We find that: (i) NCAs were inefficiently restrictive prior to the ban; (ii) the ban increased social welfare, albeit by a modest amount - on the order of one tenth of one percent; (iii) although the ban increased welfare, it was insufficient to attain the social optimum; (iv) attaining the social optimum would have required a concurrent $30 \%$ increase in the minimum wage to $\$ 10.03$ per hour (in 2006 dollars); and (v) the welfare gains from implementing this optimal policy mix would have been large - on the order of one percent.

The paper contributes to an active literature studying the effects of NCAs in low-wage labor markets. As we discuss in detail below, most of this literature is empirical in nature, seeking to measure the prevalence of NCAs in low-wage labor markets and estimating the effects of NCAs on labor turnover and wages. Our analysis complements this work in two main ways: First, we provide novel theoretical insight into the welfare implications of NCAs in general equilibrium, and study how optimal NCA policy interacts with minimum wage policy. Second, we use the model to provide explicit guidance for policy makers, in the form of a sufficient statistic that provides a practical and robust criterion for weakening NCAs, and also by showing how the model can be calibrated to produce a quantitative assessment of the welfare implications of Oregon's 2008 NCA ban for low-wage workers.

The remainder of the paper is structured as follows. In Section 2, we summarize the existing empirical literature on NCAs in low-wage labor markets. Section 3 describes the model and derives the equilibrium. In Section 4, we study the model's qualitative predictions, including the central result that NCAs increase recruitment and thus employment, and show that the model is consistent with the stylized facts discussed in Section 2. Section 5 characterizes the efficiency condition that determines the optimal level of NCAs and shows how optimal NCA policy depends on the level of the minimum wage. Finally, in Section 6, we use the model to provide quantitative evidence on the effects of NCAs, showing that, for empirically plausible parameters, NCAs are inefficient for low-wage labor markets. 


\section{NCAs in Low-Wage Labor Markets: Summary of Evidence}

While there is a long-standing literature studying NCAs for different high-skilled professions, work on the prevalence of NCAs and its effects for low-skill workers is more recent. ${ }^{8}$ The studies that do exist, however, establish clear facts that have garnered considerable attention from policy makers and the business press (see the above references). Here, we review these facts and subsequently use them to discipline our model.

\subsection{Prevalence of NCAs}

To date, we know of only two major studies - one a survey of workers and one a survey of establishments - that provide insight into the prevalence of NCAs in low-wage labor markets. The first is Starr et al. (2021), who study responses from a nationally representative survey of 11,505 labor force participants in 2014. They find that, among respondents without a bachelor's degree, $35 \%$ report having been covered by an NCA at some point in their lives while $14 \%$ report currently being covered by one. Likewise, among respondents earning under $\$ 40,000$ annually, $33 \%$ report having been covered by an $\mathrm{NCA}$ at some point and $13.3 \%$ report currently being covered by one. The authors also find that nearly $45 \%$ of respondents with less than a bachelor's degree report not knowing whether they have previously signed an NCA, whereas only $20 \%$ of respondents with at least a bachelor's degree report not knowing whether they have previously signed an NCA. Furthermore, respondents with a bachelor's degree are twice as likely to negotiate over an NCA in their contract as workers without a bachelor's degree.

The second study concerned with the prevalence of NCAs in low-wage labor markets is Colvin and Shierholz (2019), who use a national survey of human resource managers for private-sector business establishments with at least 50 employees in 2017. They find that, among firms paying average hourly wages of less than $\$ 13 /$ hour, $29 \%$ report that all employees are subject to NCAs while $38 \%$ report that some employees are subject to NCAs. These numbers increase to $31 \%$ and $57 \%$, respectively, when considering firms paying average hourly wages of between $\$ 13 /$ hour and $\$ 17 /$ hour. Similarly, the authors find that among firms whose typical employee has less than a high school diploma, $20 \%$ report that all employees are covered by NCAs and $32 \%$ report that some employees are covered. These numbers increase to $27 \%$ and $44 \%$, respectively, when considering firms whose typical employee has a high school diploma but no college.

Taken together, the two studies indicate that NCAs are commonplace in low-wage labor markets. ${ }^{9}$ Before turning to the effects of NCAs on low-wage labor markets, two observations bear emphasizing: First, because of pervasive uncertainty among workers - particularly those with limited education

\footnotetext{
${ }^{8}$ See Bishara and Starr (2016) for an excellent overview. As they write, "the only systematic evidence on the use of non-competes among workers comes from three occupations: executives (Schwab and Thomas (2006), Garmaise (2011), Bishara et al. (2015)), physicians (Lavetti et al. (2019)), and engineers (Marx (2011))." In addition, there are establishment surveys on NCAs by Kaplan and Stromberg (2003) and Galle and Koen (2001), as well as the Society of Human Resource Management. However, none of these provide sufficiently detailed or representative data to measure the prevalence of NCAs in low-wage labor markets.

${ }^{9} \mathrm{NCAs}$ are also common for low-wage workers in other countries besides the U.S. See Young (2021) for a discussion and an analysis of data for Austria.
} 
and low pay - about whether they are covered by NCAs (see discussion above), the reported prevalence of NCAs among low-wage and low-education workers almost certainly understates the true prevalence. Second, the evidence of pervasive use of no-poaching agreements among lowwage employers documented by Krueger and Ashenfelter (2018) suggests that the extent of NCAs understates the reach of mobility-restricting policies more broadly. We return to this point in Section 3 when we describe the model environment.

\subsection{Effects of NCAs}

There are very few studies to date that quantify the effects of NCAs on low-wage labor markets. Part of this is because, until the late 2010s, there were very few changes in NCA enforceability across states, and even fewer that specifically pertained to low-wage labor markets. In fact, the only instance of legislation addressing the use of NCAs for low-wage workers prior to 2017 was Oregon's 2008 ban, which is the basis for our current understanding of how NCAs affect low-wage labor markets.

In an important contribution, Lipsitz and Starr (2021) exploit Oregon's 2008 NCA ban for hourlypaid workers earning below the median family income to identify the effect of NCAs on low-wage labor markets. The authors use difference-in-difference and synthetic control designs on data from the Current Population Survey (CPS) to analyze the effects of this policy change on a variety of outcomes, including job-to-job mobility, average wages and the distribution of wages. ${ }^{10}$ Three results are particularly relevant to our analysis below: First, the authors find that the use of NCAs depresses job-to-job transitions by between $12 \%$ and $18 \%$ on average. This effect is robust to various controls and is accounted for both by job-to-job transitions within occupations and industries (65\%) but also by occupational upgrading and industry switching (35\%). Second, the authors document that the use of NCAs reduces hourly wages by between $2.2 \%$ and $3.1 \%$ on average. These changes are not offset by changes in total hours, resulting in comparable changes in weekly earnings. The authors show that these results hold across the age, wage and skill distributions, and, importantly, are stronger in occupations in which NCAs are known to be more prevalent. Finally, the authors estimate treatment effects at quantiles of the wage distribution and find that the negative wage effects of NCAs are greater farther up the wage distribution, implying that NCAs reduce wage dispersion.

These empirical results provide us with a set of stylized facts against which we compare our model's qualitative predictions (Section 4). Furthermore, we will use the estimated effects of NCAs on job-to-job transitions to calibrate our model in the quantitative application (Section 6).

\section{Model}

We study the role of NCAs in the context of an oligopsonistic model of the labor market featuring endogenous recruitment decisions by firms. Specifically, we augment a model of labor turnover in

\footnotetext{
${ }^{10}$ The authors also consider the implications of NCAs for occupational upgrading, the share of salaried workers, and weekly hours worked.
} 
the spirit of Burdett and Mortensen (1998) with a model of recruitment that generalizes the model studied in Manning (2003) by allowing the rate at which employed and non-employed workers receive job offers to differ. This distinction is critical for studying the role of NCAs, which principally constrain job-to-job transitions, and thus differentially affect employed and non-employed workers.

\subsection{Environment}

Time is continuous and discounted at rate $r$. The economy is populated by a unit measure of identical firms and a unit measure of identical workers who are either employed at a firm or non-employed. There is a single consumption good and workers derive linear utility from consumption. Each firm produces the consumption good with technology $y=p l$, where $p$ denotes a firm's productivity and $l$ denotes the measure of workers employed at the firm. Workers receive wage $w$ when employed with a given firm and flow utility $b$ when not employed. Workers also receive dividends from a perfectly diversified portfolio of firm ownership.

\subsubsection{Search, matching, and NCAs}

Search is sequential and undirected. Both non-employed and employed workers search. Nonemployed workers receive job offers from firms at rate $\lambda^{n}(Z)$, where $Z$ is a measure of average recruitment intensity as defined below, and $\lambda^{n}(Z)$ is strictly increasing and weakly concave in $Z$ with $\lambda^{n}(0)=0$. Employed workers receive job offers from new firms at rate $\lambda^{e}(Z ; \gamma)$, where once again $\lambda^{e}(Z ; \gamma)$ is strictly increasing and weakly concave in $Z$ with $\lambda^{e}(0 ; \gamma)=0$, and $\gamma \in[0,1]$ is a parameter that reflects restrictions on job-to-job transitions imposed by NCAs as described below. Matches are destroyed at the exogenous rate $\delta$.

The dependence of $\lambda^{e}(Z ; \gamma)$ on the parameter $\gamma$ is intended to capture, in a flexible way, the role of NCAs as a source of monopsony power for firms. Reflecting this, we make the following assumptions:

A1. $\lambda^{e}(Z ; \gamma)$ is strictly decreasing in its second argument $\left(\lambda_{\gamma}^{e}<0\right)$

A2. $\lim _{\gamma \rightarrow 0} \lambda^{e}(Z ; \gamma) \in(0, \infty)$ for any $Z>0$

A3. $\lim _{\gamma \rightarrow 1} \lambda^{e}(Z ; \gamma)=0$

A1 implies that the direct effect of higher values of $\gamma$ is to reduce the rate at which employed workers receive offers from new employers and thus increase firms' ability to extract surplus from employment relationships; ${ }^{11}$ A2 reflects that NCAs are not the only source of frictions impeding job-to-job transitions in the model, and thus are not the only source of monopsony power; and A3 reflects that, in principle, sufficiently pervasive and enforceable NCAs could fully eliminate job-to-job transitions. We view these assumptions, particularly A2 and A3, as reflecting the most natural characterization of the scope of NCA policy, broadly defined, in low-wage labor markets.

By modeling NCAs via the parameter $\gamma$ as described above, we are abstracting from details of the contracting environment that support the existence of NCAs as an equilibrium outcome. Instead, to

\footnotetext{
${ }^{11}$ As we discuss at length below, there is also a general equilibrium effect, since $\gamma$ affects equilibrium recruitment $Z$, which in turn directly affects $\lambda^{e}(Z ; \gamma)$.
} 
analyze the efficiency questions that are the focus of this paper, we think of $\gamma$ as a policy parameter that, similar to a minimum wage, a government has at its disposal to influence equilibrium outcomes. Another virtue of modeling NCAs through $\gamma$ is that doing so affords flexibility with respect to interpretation, thus allowing us to focus on the equilibrium implications of a broad class of mobilityrestricting policies. For example, $\gamma$ can be interpreted as reflecting several of the constituent components of the Bishara (2011) NCA enforceability index, such as how an employer's protectable interest is defined, the burden of proof required of the plaintiff, whether overly broad contracts can be rewritten or must be thrown out entirely, and so on. In fact, because we do not take a stance on the nature of the contracting environment, our framework is sufficiently general to allow $\gamma$ to be interpreted even more broadly as encompassing other entirely distinct classes of mobility-restricting policies, such as no-poach agreements between firms. ${ }^{12}$

It is also worth highlighting that, in our setup, offer rates for employed and non-employed workers are generally different from each other; i.e. $\lambda^{n}(Z) \gtreqless \lambda^{e}(Z ; \gamma)$. Even if $\gamma=0$ (no NCAs), there is no assumption that the two rates need to be the same, which is consistent with evidence from Faberman et al. (2017). In addition to our focus on NCA policy, this is an important distinguishing feature of our environment relative to the one presented in Manning (2003). As we show below, this distinction has important implications for efficiency and optimal policy.

\subsubsection{Wage determination}

Firms post fixed wage contracts such that all workers receive the same wage for the duration of the employment relationship. Because of our maintained focus on low-wage labor markets, we assume that posted wages cannot be below minimum wage $w_{\min }$, which may or may not be binding in equilibrium depending on the parameterization. We use $G(w)$ to denote the cumulative density function of the wage distribution across workers employed by the different firms, and $H(w)$ the cumulative density function of the sampling or offer distribution, with corresponding densities $g(w)$ and $h(w)$.

\subsubsection{Recruitment intensity}

Firms can influence the arrival of new potential hires through recruitment intensity $z$ at flow cost $c(z)$. In general, the optimal choice of recruitment intensity will depend on the firm's wage: $z=z(w)$. We therefore express average recruitment intensity as

$$
Z=\int z(w) d H(w)
$$

Following Manning (2003), we assume that a firm choosing recruitment intensity $z$ receives a share $z / Z$ of all matches with new potential hires. The cost function is assumed to be strictly increasing and strictly convex in $z$ with $c(0)=c^{\prime}(0)=0$ so as to ensure an interior solution to the firm's

\footnotetext{
${ }^{12}$ Krueger and Ashenfelter (2018) document that $58 \%$ of major franchise chains include no-poach clauses in their franchise contracts.
} 
recruitment problem. As discussed in Manning (2003), $c(z)$ may be thought of as encompassing a range of recruitment costs - not only advertising costs but also the administrative costs associated with the application and hiring process as well as training costs. In Appendix E, we show that all of our qualitative conclusions continue to hold in an otherwise identical model with free entry of firms instead of endogenous recruitment decisions. ${ }^{13}$

\subsection{Workers}

We begin by analyzing workers' reservation wages. Conditional on average recruitment intensity $Z$ and NCA parameter $\gamma$ (which only operate through $\lambda^{n}(Z)$ and $\lambda^{e}(Z ; \gamma)$ ), the analysis is identical to that in Burdett and Mortensen (1998).

Upon arrival of a job offer at wage $w$, a non-employed worker decides whether or not to accept the offer. Suppressing dependence of value functions on $Z$ for ease of notation, the flow value of rejecting the offer is given by

$$
r V^{n}=b+\lambda^{n}(Z) \int \max \left\{V^{e}\left(w^{\prime}\right)-V^{n}, 0\right\} d H\left(w^{\prime}\right)
$$

and the flow value of accepting the offer is

$$
r V^{e}(w)=w+\lambda^{e}(Z ; \gamma) \int \max \left\{V^{e}\left(w^{\prime}\right)-V^{e}(w), 0\right\} d H\left(w^{\prime}\right)-\delta\left[V^{e}(w)-V^{n}\right] .
$$

Because the value of accepting an offer is increasing in $w$ while the value of rejecting the offer is invariant to $w$, the optimal search strategy takes the form of a reservation wage $R$ such that $V^{e}(R)=V^{n}$. Evaluating (3) at $R$ and using (2), we obtain the following characterization of the reservation wage

$$
\begin{aligned}
R & =b+\left(\lambda^{n}(Z)-\lambda^{e}(Z ; \gamma)\right) \int_{R}^{\infty}\left[V^{e}\left(w^{\prime}\right)-V^{e}(R)\right] d H\left(w^{\prime}\right) \\
& =b+\left(\lambda^{n}(Z)-\lambda^{e}(Z ; \gamma)\right) \int_{R}^{\infty}\left[\frac{1-H\left(w^{\prime}\right)}{r+\delta+\lambda^{e}(Z ; \gamma)\left(1-H\left(w^{\prime}\right)\right)}\right] d H\left(w^{\prime}\right)
\end{aligned}
$$

Employed workers, in turn, accept any new job offer that is above the current wage and reject all other offers since jobs differ only in the wage paid and the current wage is, by assumption, fixed.

In what follows, it will be useful to define a wage floor $\underline{w}$ as the greater of the reservation wage and the minimum wage

$$
\underline{w}=\max \left\{R, w_{\min }\right\} .
$$

We say that the minimum wage is binding if $w_{\min }>R$ and non-binding otherwise. Without loss of generality, we assume that even if the minimum wage is non-binding, firms do not post wage offers below the reservation wage since such wage offers would be automatically rejected by potential hires.

\footnotetext{
${ }^{13}$ One can think of the distinction between our model and one with free entry as a distinction between incorporating an intensive versus an extensive margin of job creation, hence the qualitative similarity.
} 
Hence, $\underline{w}$ is the lower bound of the wage and offer distributions $G(w)$ and $H(w)$.

\subsection{Firms}

Firms optimally choose a wage offer, $w$, and recruitment intensity, $z$, to maximize steady-state flow profits $^{14}$

$$
\pi(w, z)=(p-w) l(w, z)-c(z),
$$

where $l(w, z)$ is the steady-state quantity of labor available to a firm offering wage $w$ and exerting recruitment intensity $z$. To characterize $l(w, z)$, note that in steady state, a constant distribution of workers across firms requires that the inflow of workers to a firm offering wage $w$ must equal the outflow of workers from that firm. Denoting by $r(w, z)$ the total inflow of new hires and by $s(w)$ the separation rate for a firm with wage $w$, this implies that $r(w, z)=l(w, z) s(w)$, or equivalently

$$
l(w, z)=\frac{r(w, z)}{s(w)} .
$$

The total inflow of new hires, in turn, equals the sum of hires from other firms, given by $r^{e}(w, z)=$ $(z / Z) \lambda^{e}(Z ; \gamma) e G(w)$, and the hires from non-employment, given by $r^{n}(z)=(z / Z) \lambda^{n}(Z)(1-e)$, where $e$ denotes the fraction of employed workers, defined in steady state as ${ }^{15}$

$$
e=\frac{\lambda^{n}(Z)}{\delta+\lambda^{n}(Z)} .
$$

Thus, we have,

$$
r(w, z)=r^{e}(w, z)+r^{n}(z)=\frac{z}{Z}\left[\lambda^{e}(Z ; \gamma) e G(w)+\lambda^{n}(Z)(1-e)\right] .
$$

The separation rate, in turn, equals the sum of the match destruction rate and the rate at which workers receive outside offers multiplied by the probability that an outside offer dominates the current wage

$$
s(w) \equiv \delta+\lambda^{e}(Z ; \gamma)(1-H(w))
$$

Substituting (10) and (11) into (8), then substituting (8) into (7), and finally defining the labor supply to a firm choosing average recruitment intensity (i.e. choosing $z=Z$ ) as

$$
l(w) \equiv \frac{\lambda^{e}(Z ; \gamma) e G(w)+\lambda^{n}(Z)(1-e)}{\left[\delta+\lambda^{e}(Z ; \gamma)(1-H(w))\right]}
$$

\footnotetext{
${ }^{14}$ The problem of maximizing steady-state flow profits is equivalent to the dynamic problem of maximizing the expected present discounted value of profits under the assumption that $r / \lambda^{n} \rightarrow 0, r / \lambda^{e} \rightarrow 0$, and $r / \delta \rightarrow 0$, as is commonly assumed in the literature. See Manning (2006) for a formal demonstration of this point.

${ }^{15}$ For notational simplicity, we suppress dependence of equilibrium objects on average recruitment intensity $Z$ and equilibrium wage and offer distributions $G(w)$ and $H(w)$. We continue to express $\lambda^{n}(Z)$ and $\lambda^{e}(Z ; \gamma)$ as functions of $Z$ to highlight that $Z$ operates directly through these variables.
} 
we can rewrite the firm's steady-state flow profits as

$$
\pi(w, z)=(p-w) \frac{z}{Z} l(w)-c(z) .
$$

Equation (13) highlights that the labor supply available to a firm, and therefore its profit gross of recruitment costs, is linear in its recruitment effort. This property allows for a straightforward characterization of both the optimal choice of $w$ and $z$.

Maximizing (13) with respect to the wage $w$ yields the first-order condition

$$
\frac{l^{\prime}(w)}{l(w)}=(p-w)^{-1} \quad \text { for } w \geq \underline{w} .
$$

This is a first-order differential equation in $l(w)$, which can be solved by integrating both sides of the equation and exponentiating, giving ${ }^{16}$

$$
l(w)=\frac{e^{k}}{p-w},
$$

where $k$ is some constant of integration. This solution has several important implications. First, it implies that the supply of labor available to a firm, $l(w, z)$, is increasing in $w$. Second, it implies that wages lie strictly below the marginal product of labor, $p$. Third, it implies that a firm's optimal wage does not depend on its recruitment intensity, $z$. This last implication follows from the linearity of $l(w, z)$ in $z$, which allowed us to rewrite the profit function in (7) as (13). Plugging (15) back into (13), we obtain immediately that firm profits are independent of the wage. Hence, firms are indifferent between all wages in the support of the offer distribution (the upper bound of which we solve for below), which is the celebrated result of equilibrium wage dispersion in wage-posting models developed by Burdett and Mortensen (1998).

Maximizing (13) with respect to $z$ yields the first-order condition

$$
\frac{(p-w) l(w)}{Z}-c^{\prime}(z)=0 .
$$

The important observation here is that, because profits gross of recruitment costs are linear in a firm's recruitment effort $z$ (per equation (13)), the marginal benefit of recruitment to a firm is independent of its level of recruitment. This immediately implies that all firms choose the same recruitment intensity in equilibrium; i.e. $z=Z$ for all firms. ${ }^{17}$ One of the implications of this is that, in equilibrium, all firms earn equal profits and thus - conditional on average recruitment $Z$ and the implied values of $\lambda^{e}(Z ; \gamma)$ and $\lambda^{n}(Z)$ - the model is identical to Burdett and Mortensen $(1998) .^{18}$

\footnotetext{
${ }^{16}$ Integrating both sides yields $\ln (l(w))=-\ln (p-w)+k$, which can be exponentiated to give the result in (15).

${ }^{17}$ Substituting (15) into (16) yields $c^{\prime}(z)=e^{k} / Z$. Strict convexity of $c(\cdot)$ then implies that there is a unique solution for $z$ that does not depend on $w$. Furthermore, $c^{\prime}(0)=0$ implies that $z=Z=0$ cannot be an equilibrium. See Manning (2003) for a similar argument when offer rates are identical for employed and non-employed workers.

${ }^{18}$ The result follows immediately from the preceding observations about wages and recruitment: Substituting (15)
} 
Letting $z=Z$ and using (12), we can write (16) as

$$
\underbrace{c^{\prime}(Z)}_{\text {Marg. cost }}=\underbrace{\frac{p-w}{\delta+\lambda^{e}(Z ; \gamma)(1-H(w))}}_{\text {PDV of new hire }} \cdot[\underbrace{\frac{e \lambda^{e}(Z ; \gamma) G(w)}{Z}}_{\begin{array}{c}
\text { Additional hires } \\
\text { (poached) }
\end{array}}+\underbrace{\frac{(1-e) \lambda^{n}(Z)}{Z}}_{\begin{array}{c}
\text { Additional hires } \\
\text { (from unempl.) }
\end{array}}]
$$

where $e$ is the employment rate defined in (9). Firms equate the marginal cost of recruitment with the expected marginal benefit. The latter is the additional inflow of new hires associated with the extra recruitment effort - including hires from other firms and from unemployment-multiplied by the expected present discounted value of flow profits generated by each of those hires. ${ }^{19}$

\subsection{Equilibrium}

We are now prepared to characterize the steady-state equilibrium of the economy. First, note that in steady state, inflows into jobs paying wages less than $w$ must be equal to outflows from such jobs, so that $\lambda^{n}(Z)(1-e) H(w)=e G(w)\left[\delta+\lambda^{e}(Z ; \gamma)(1-H(w))\right]$. Using (9) to eliminate $e$, we can solve for the distribution of wages across workers, $G(w)$ :

$$
G(w)=\frac{H(w)}{1+\lambda^{e}(Z ; \gamma) / \delta(1-H(w))}
$$

Next, using (5), (14), (18), and the fact that $H^{\prime}(w) l(w)=G^{\prime}(w) e$ (both sides are expressions for the mass of employees paid wage $w$ ), we can solve for the unique distribution of wage offers, $H(w):{ }^{20}$

$$
H(w)=\frac{1+\lambda^{e}(Z ; \gamma) / \delta}{\lambda^{e}(Z ; \gamma) / \delta}\left[1-\left(\frac{p-w}{p-\underline{w}}\right)^{\frac{1}{2}}\right] \quad \text { for } w \in[\underline{w}, \bar{w}]
$$

where

$$
\begin{aligned}
& \underline{w}=\max \left\{R, w_{\min }\right\} \\
& R=\frac{b\left(\delta+\lambda^{e}(Z ; \gamma)\right)^{2}+p\left(\lambda^{n}(Z)-\lambda^{e}(Z ; \gamma)\right) \lambda^{e}(Z ; \gamma)}{\left(\delta+\lambda^{e}(Z ; \gamma)\right)^{2}+\left(\lambda^{n}(Z)-\lambda^{e}(Z ; \gamma)\right) \lambda^{e}(Z ; \gamma)} \\
& \bar{w}=p-(p-\underline{w})\left(\frac{1}{1+\lambda^{e}(Z ; \gamma) / \delta}\right)^{2} .
\end{aligned}
$$

Finally, profit equalization implies that we can evaluate (17) at $w=\underline{w}$ without loss of generality. Then, using (9) to eliminate $e$ and using (20) and (21) to write $\underline{w}$ as an explicit function of $Z$ for a given level of NCAs and the minimum wage $\left(\underline{w}=\underline{w}\left(Z ; \gamma, w_{\min }\right)\right)$, (17) defines an implicit

into (13) and noting that all firms choose the same $z$, it must be that all firms earn the same profit in equilibrium.

${ }^{19}$ Note that, because all firms recruit with intensity $Z$ and earn equal profits, we can write the first-order condition in (17) in terms of any wage in the support of the offer distribution. We will exploit this fact below.

${ }^{20}$ See Appendix A.1 for complete derivations of (19), (21) and (22). 
function relating equilibrium recruitment intensity to the level of NCAs and the minimum wage, $Z=Z\left(\gamma, w_{\min }\right)$ :

$$
c^{\prime}(Z)=\frac{p-\underline{w}\left(Z ; \gamma, w_{\min }\right)}{\delta+\lambda^{e}(Z ; \gamma)} \frac{\delta \lambda^{n}(Z)}{Z\left(\delta+\lambda^{n}(Z)\right)} .
$$

The decentralized equilibrium is thus defined as the solution $\{G(w), H(w), \underline{w}, R, \bar{w}, Z\}$ to (18)-(23).

That the decentralized equilibrium is unique follows from the fact that (18)-(22) can be used to express $G(w), H(w), \underline{w}, R, \bar{w}$ as explicit functions of $Z$ (see Appendix A.1 for details), together with the fact that convexity of $c(\cdot)$ implies that there is a unique value of $Z$ that solves (23) (see Appendix A.3 for details).

We next turn our attention to how NCAs affect equilibrium recruitment intensity - and thus other endogenous variables of interest - through the implicit solution $Z=Z\left(\gamma, w_{\min }\right)$ to $(23) .{ }^{21}$

\section{Effects of NCAs}

This section studies the qualitative effects of NCAs through the lens of the model developed in Section 3. We begin by analyzing how NCAs affect recruitment and offer rates. The main result is that NCAs necessarily increase recruitment and therefore offer rates for non-employed workers. An immediate corollary of this finding is that NCAs must also increase employment, a result that will be critical to our welfare analysis in Sections 5 and 6 . We then show that, despite increasing recruitment, NCAs necessarily reduce offer rates for employed workers. This implies that NCAs reduce job-to-job transitions, average wages, and wage dispersion, all of which are consistent with the observed effects of NCAs in low-wage labor markets surveyed in Section 2. Furthermore, as we show, these results all hold regardless of whether or not there is a binding minimum wage.

\subsection{Recruitment and offer rates}

The effect of NCAs on recruitment is not obvious ex ante. On the one hand, NCAs make it more difficult for firms to poach workers from other firms, thereby lowering the return to recruitment effort. On the other hand, NCAs reduce turnover and thus increase the average length of time for which a worker will continue to generate revenue at a firm. Proposition 1 clarifies the nature of this relationship.

Proposition 1 (Recruitment). NCAs increase recruitment intensity.

Proof. See Appendix B.

Intuitively, since all firms choose the same recruitment intensity, the effect of NCAs on average recruitment must be the same as the effect of NCAs on recruitment of firms offering the lowest wage $\underline{w}$. But because such firms are unable to poach workers from firms offering higher wages, the negative poaching-deterrent effect of NCAs is irrelevant for them. Thus, the only effect of NCAs for

\footnotetext{
${ }^{21}$ See Appendix B.3 for an analysis of how changes in the minimum wage affect recruitment intensity.
} 
such firms is to increase retention, which stimulates recruitment. As we will see, this result is at the heart of our model's implications for the efficiency of NCAs.

From Proposition 1, it follows immediately that NCAs increase offer rates for non-employed workers, $\lambda^{n}(Z)$. In contrast, the effect of NCAs on offer rates for employed workers, $\lambda^{e}(Z ; \gamma)$, is a priori ambiguous: NCAs directly reduce job offers through $\gamma$, but also increase job offers indirectly because of their positive equilibrium effect on recruitment intensity $Z$. Proposition 2 disentangles the two forces.

Proposition 2 (Offer rates). NCAs reduce the rate at which employed workers receive offers from potential new employers.

Proof. See Appendix B.

To understand this result, suppose stricter enforcement of NCAs increased aggregate recruitment so much that it resulted in an increase in the offer rate for employed workers despite directly restricting their mobility. In this case, the increased offer rate would cause new hires to be poached more quickly, thereby depressing the return to hiring and firms' individual incentives to recruit. This would result in firms reducing their recruitment intensity, implying, in turn, that the conjectured increase in aggregate recruitment cannot occur in equilibrium.

\subsection{Employment}

An immediate consequence of the effects of NCAs on recruitment and thus offer rates of non-employed workers established in Proposition 1 is that NCAs must also increase employment.

Proposition 3 (Employment). NCAs increase employment.

Proof. By Proposition 1, NCAs increase recruitment, $Z$, and thus $\lambda^{n}(Z)$. The result then follows from the expression for equilibrium employment in $(9), e=\frac{\lambda^{n}(Z)}{\delta+\lambda^{n}(Z)}$, which is increasing in $\lambda^{n}(Z)$.

The result implies that the welfare implications of NCAs are fundamentally ambiguous. On the one hand, NCAs stimulate recruitment and employment. On the other hand, NCAs stimulate recruitment and lead to congestion in labor markets. Which force prevails, and under what conditions, should be a key concern for policy makers - and is thus the focus of Sections 5 and 6 of this paper. Before turning to efficiency, however, we investigate whether our model is consistent with the effects of NCAs in low-wage labor markets documented in Lipsitz and Starr (2021).

\subsection{Mobility and wages}

As discussed in Section 2, the existing empirical literature on NCAs in low-wage labor markets has found that NCAs reduce job-to-job transitions, depress average wages, and compress the wage distribution. Proposition 4 summarizes our model's implications for these variables. See Appendix B for all derivations. 
Proposition 4 (Mobility and wages). NCAs reduce the average rate of job-to-job transitions, reduce average wages, and reduce wage dispersion (as measured by the mean-min wage ratio).

Proof. See Appendix B.

Thus, our model is consistent with the empirical findings in Starr et al. (2021) concerning the effects of NCAs on low-wage labor markets. Below, we provide further discussion of these results.

\subsubsection{Job-to-job transitions}

Consider first the average rate of job-to-job transitions. Because NCAs reduce the offer rate of employed workers, and the average rate of job-to-job transitions is increasing in this offer rate, it follows that NCAs unambiguously reduce the average rate of job-to-job transitions, as in the data. This can be seen formally by noting that in the model, the average rate of job-to-job transitions, which we will denote by $\chi$, is given by

$$
\begin{aligned}
\chi & \equiv \int_{\underline{w}}^{\bar{w}} \lambda^{e}(Z ; \gamma)(1-H(w)) d G(w) \\
& =\delta\left[\frac{\delta+\lambda^{e}(Z ; \gamma)}{\lambda^{e}(Z ; \gamma)} \ln \left(\frac{\delta+\lambda^{e}(Z ; \gamma)}{\delta}\right)-1\right] .
\end{aligned}
$$

Inspection of (25) bears out the preceding intuition that the average rate of job-to-job transitions is increasing in the offer rate of employed workers. As we see in (25), this is true regardless of whether or not the minimum wage is binding. The first result in Proposition 4 then follows immediately from Proposition 2.

\subsubsection{Average wage}

The effect of NCAs on the average wage is more complicated because NCAs affect the equilibrium wage distribution through three channels. To see this, write the average wage in the model as

$$
\begin{aligned}
E[w] & \equiv \int_{\underline{w}}^{\bar{w}} w d G(w) \\
& =\underline{w}+\int_{\underline{w}}^{\bar{w}}(1-G(w)) d w .
\end{aligned}
$$

Inspection of (27) reveals that NCAs potentially impact the average wage by changing (i) the distribution of wages across workers, $G$, (ii) the highest wage offered by firms, $\bar{w}$, and (iii) the lowest wage offered by firms, $\underline{w}$ (provided the minimum wage is non-binding). To understand why NCAs depress wages, it is instructive to consider the case of a binding minimum wage, in which case only the first two channels are operative. In this case, because NCAs reduce the offer rate of employed workers, workers climb the job ladder more slowly, resulting in a leftward shift in the distribution of wages, $G$, and, consequently, a reduction in the maximum attainable wage $\bar{w}$. Both of these effects work to depress average wages via (27). In the absence of a binding minimum wage, however, NCAs 
will also typically (although not necessarily) increase reservation wages: Strict NCA enforcement means that workers find jobs from unemployment more quickly (due to increased recruitment) and are stuck with those jobs for longer (due to a lower rate of job-to-job transitions), and will thus be inclined to wait for a good offer, resulting in higher reservation wages. As the second result in Proposition 4 clarifies, however, this effect is never strong enough to dominate the other two effects described above.

\subsubsection{Wage dispersion}

Finally, we consider the effect of NCAs for wage dispersion, as measured by the mean-min wage ratio. In the case of a binding minimum wage, the lowest wage in the economy is the minimum wage, so the effect of NCAs on the mean-min wage ratio is proportional to their effect on average wages. Thus, because NCAs necessarily reduce average wages, they also necessarily reduce wage dispersion. In the case of a non-binding minimum wage, observe that we can write the mean-min wage ratio as

$$
\begin{aligned}
M m & \equiv E[w] / \underline{w} \\
& =\frac{1+\frac{\lambda^{n}-\lambda^{e}}{\delta+\lambda^{e}}}{\rho+\frac{\lambda^{n}-\lambda^{e}}{\delta+\lambda^{e}}}
\end{aligned}
$$

where $\rho \equiv b / E[w]$ is the replacement rate - the ratio of the flow value of non-employment to the average wage. Because NCAs depress average wages (and thus increase $\rho$ ) and also drive a wedge between the offer rates of non-employed workers and employed workers (and thus increase $\frac{\lambda^{n}-\lambda^{e}}{\delta+\lambda^{e}}$ ), inspection of (29) reveals that if $\rho<1$, NCAs must reduce the mean-min wage ratio. On the other hand, if $\rho>1$ so that average wages are less than the flow value of non-employment $(E[w]<b)$, it must be that the lowest wage in the economy — non-employed workers' reservation wage - is also less than the flow value of non-employment $(R<b)$, which is only optimal for workers if they get to sample offers more quickly during employment than non-employment $\left(\lambda^{e}>\lambda^{n}\right)$. But this implies that NCAs increase the reservation wage, and if NCAs increase the reservation wage and reduce the average wage, then NCAs must reduce the mean-min ratio (which, absent a binding minimum wage, is just the ratio of these two variables, $E[w] / R$ ). This is the third result in Proposition 4 .

Thus, we conclude that regardless of whether or not the minimum wage is binding, our model is consistent with the stylized facts set out in Section 2. We next turn to studying the efficiency implications of NCAs in low-wage labor markets.

\section{Optimal NCA Policy and the Minimum Wage}

To formulate optimal NCA policy, we start by deriving the constrained-efficient allocation and show that efficiency of the decentralized economy is characterized by a condition similar to the familiar Hosios condition in random search models with bargaining. The condition implies that, depending on the combination of NCA enforceability $(\gamma)$ and the level of the minimum wage $\left(w_{\min }\right)$, 
the decentralized equilibrium can feature either excessive recruitment or insufficient recruitment, thus providing scope for NCA policy to improve welfare. We show that optimal NCA policy depends crucially on the level of the minimum wage. Furthermore, we show that, in general, optimal NCA policy alone is not sufficient to guarantee that the efficient allocation can be achieved. Likewise, optimal minimum wage policy alone is not sufficient to guarantee efficiency. Critically, however, we show that there always exists a combination of NCA and minimum wage policies that implements the efficient allocation.

\subsection{Planner's problem}

The social planner chooses average recruitment intensity $Z$ to maximize total output net of recruitment costs, taking as given the underlying matching technology. Making explicit the dependence of the level of employment on recruitment intensity, $e=e(Z)$, the social planner chooses recruitment intensity to maximize

$$
\Omega(Z)=e(Z) p+(1-e(Z)) b-c(Z)
$$

subject to the flow equation for employment, which in steady state is given by (9). The constrainedefficient allocation $Z^{S P}$ therefore satisfies

$$
c^{\prime}(Z)=e^{\prime}(Z)(p-b) .
$$

The planner equates the marginal social benefit of recruitment - the increase in employment multiplied by the net gain in output from moving a worker from non-employment to employment, $e^{\prime}(Z)(p-b)$ - with the marginal social cost of recruitment, $c^{\prime}(Z)$.

\subsection{A Hosios-type condition}

Comparison of the social optimum in (31) with the firm's first-order condition in (23) shows that, because the marginal cost of recruitment for a firm is the same as for the planner, the decentralized equilibrium is efficient when the private and social marginal benefit of recruitment coincide. ${ }^{22}$ Combining the two equations, defining $\epsilon_{Z}^{e}(Z)$ as the elasticity of employment with respective to aggregate recruiting intensity $Z, \Pi^{\mathrm{SP}} \equiv \frac{p-b}{\delta}$ as the social present discounted value of a match, and $\Pi(\underline{w}(Z) ; Z) \equiv \frac{p-\underline{w}(Z)}{\delta+\lambda^{e}(Z ; \gamma)}$ as the private present discounted value of a match for a firm paying wage $w=\underline{w}$, we obtain the following efficiency condition:

Proposition 5 (Efficiency). The decentralized equilibrium is efficient, i.e. $Z=Z^{S P}$, if and only if

$$
\frac{1}{\epsilon_{Z}^{e}(Z)}=\frac{\Pi^{S P}}{\Pi(\underline{w}(Z) ; Z)}
$$

There is excess recruitment $\left(Z>Z^{S P}\right)$ in the decentralized equilibrium if $\epsilon_{Z}^{e}(Z)<\frac{\Pi(\underline{w}(Z) ; Z)}{\Pi^{S P}}$ and there is insufficient recruitment $\left(Z<Z^{S P}\right)$ in the decentralized equilibrium if $\epsilon_{Z}^{e}(Z)>\frac{\Pi(\underline{w}(Z) ; Z)}{\Pi^{S P}}$.

\footnotetext{
${ }^{22}$ See footnote 24 and Appendix $\mathrm{C}$ for a discussion of using (17) rather than (23) to derive the efficiency condition.
} 
Proof. See Appendix C.

This condition reflects two forces affecting the decentralized equilibrium. The left-hand side reflects a tendency towards excessive recruitment because of congestion externalities in firms' recruitment decisions: From the perspective of an individual firm taking $Z$ as given, employment is linear in recruitment effort $z$, which implies an elasticity of firm-specific employment with respect to recruitment of 1 . By contrast, from the perspective of the planner, aggregate employment $e(Z)$ is concave in $Z$, with an elasticity of $\epsilon_{Z}^{e}(Z)<1$. As a consequence, there is a tendency for firms to recruit more than is socially optimal, and the extent of this tendency is measured by the ratio of the two elasticities, which appears on the left-hand side of $(32){ }^{23}$

On the other hand, the right-hand side of (32) reflects a tendency towards insufficient recruitment resulting from the presence of job-to-job transitions, which implies that the private and social values of a match are not generally the same. This happens because (i) poaching causes matches to be destroyed at a faster rate than they otherwise would be $\left(\delta+\lambda^{e}(Z ; \gamma)>\delta\right)$, leading firms to discount the flow value of a match at a higher rate than the planner, and (ii) the wage paid by firms need not be equal to the social opportunity cost of employment $(\underline{w} \lesseqgtr b)$, implying that the flow value of a match is not necessarily the same for private firms and the planner. The totality of these two forces always results in a tendency towards under-recruitment in the decentralized equilibrium because both arise due to the presence of job-to-job transitions (i.e. $\lambda^{e}(Z ; \gamma)>0$ ), which erode firms' wage-setting power and thus make matches, and so recruitment, less profitable than they would be in the case of pure monopsony (in which case all firms offer $w=R=b$ and the private value of a match coincides with that of the planner: $\left.\lambda^{e}(Z ; \gamma) \rightarrow 0 \Longrightarrow \Pi(\underline{w}(Z) ; Z)=\Pi^{S P}=\frac{p-b}{\delta}\right)$. Efficiency requires that these competing forces exactly offset each other in equilibrium. ${ }^{24}$

Condition (32) is similar in spirit to the well-known "Hosios condition" in random search models with wage bargaining (Hosios, 1990). In such models, efficiency requires that the elasticity of the match function with respect to vacancies is exactly equal to the firm's share of the match surplus (given by one minus the workers' bargaining power). In effect, when workers have lower bargaining power, the value of a match is greater for firms, which stimulates entry and (eventually) pushes the decentralized equilibrium into a region with inefficiently high employment. In the present context, NCAs have an analogous effect to limiting workers' bargaining power in bargaining models - both promote job creation, the desirability of which depends on the nature of the underlying matching

\footnotetext{
${ }^{23}$ The elasticity of steady-state employment $e(Z)$ with respect to average recruitment intensity $Z$ is given by $\epsilon_{Z}^{e}(Z)=(1-e(Z)) \epsilon_{Z}^{\lambda^{n}}$, where $\epsilon_{Z}^{\lambda^{n}}$ denotes the elasticity of the offer rate $\lambda^{n}(Z)$ for non-employed workers with respect to $Z$. This elasticity reflects that the planner takes into account both the direct effect of $Z$ on $\lambda^{n}(Z)$ and the indirect effect of $Z$ on $e(Z)$ via its effect on $\lambda^{n}(Z)$.

${ }^{24}$ There is a third source of inefficiency that affects recruitment of firms choosing $w>\underline{w}$ : Unproductive poaching of already-employed workers. To see this, note that we can write the condition in (32) using the more general first-order condition in (17) rather than the special case $(w=\underline{w})$ in $(23)$. This yields the efficiency condition $\frac{1+\phi(w ; Z)}{\epsilon_{Z}^{e}(Z)}=\frac{\Pi^{\mathrm{SP}}}{\Pi(w ; Z)}$ for $w \in[\underline{w}, \bar{w}]$, where $\phi(w ; Z) \equiv \frac{r^{e}(w, Z)}{r^{n}(Z)}$ is the ratio of hires poached from employment to hires from non-employment. Note that the special case embodied in $(32)$ corresponds to $\phi(\underline{w} ; Z)=0$. Importantly, profit-equalization and the fact that all firms choose the same $z=Z$ in equilibrium implies that these conditions have identical implications for efficiency. See Appendix $C$ for a derivation of the general case described here.
} 
frictions. It follows that condition (32) provides scope for NCA policy to improve welfare.

Condition (32) also highlights the interaction between NCA and minimum wage policy in our model. Recall from our characterization of the decentralized equilibrium that the solution to (23) can be written as $Z=Z\left(\gamma, w_{\min }\right)$ where, in the presence of a binding minimum wage, $d Z / d w_{\min }<0$ : A higher value of the minimum wage in this situation depresses the private gain from a match, $\Pi(\underline{w}(Z) ; Z)$, thereby reducing recruitment. ${ }^{25}$ As such, a higher minimum wage acts as a substitute for weaker enforcement of NCAs, implying that optimal NCA policy depends crucially on the level of the minimum wage.

\subsection{Optimal NCA policy}

To characterize optimal NCA policy as a function of the minimum wage, $\gamma^{*}\left(w_{\min }\right)$, we consider two cases. The first case is depicted in Figure $2 \mathrm{a}$ and assumes that the environment is such that $Z=Z(0,-\infty)>Z^{S P}$; i.e. without NCAs or a minimum wage, the decentralized equilibrium is characterized by excess recruitment. In this case, optimal NCA policy is characterized by $\gamma^{*}(-\infty)=0$ since any imposition of NCAs would increase recruitment and thus reduce welfare. As the minimum wage increases and becomes binding, recruitment declines until we reach some threshold minimum wage, $w_{\min }^{L}$, for which $Z=Z\left(0, w_{\min }^{L}\right)=Z^{S P}$. For minimum wage levels above this threshold, optimal NCA policy is interior and increasing in the minimum wage (i.e. $\gamma^{*}\left(w_{\min }\right)>0$ with $\frac{\partial \gamma^{*}\left(w_{\min }\right)}{\partial w_{\min }}>0$ for $\left.w_{\min }>w_{\min }^{L}\right)$ and achieves efficiency until NCA policy reaches its limit, $\gamma^{*}\left(w_{\min }^{H}\right)=1$. Beyond this point, any further increase in the minimum wage will lower recruitment below the welfare-maximizing level.

The second case is depicted in Figure $2 \mathrm{~b}$ and assumes that the environment is such that $Z=$ $Z(0,-\infty)<Z^{S P}$; i.e. without NCAs or a minimum wage, the decentralized equilibrium results in insufficient recruitment. In this case, optimal NCA policy is characterized by $\gamma^{*}(-\infty)>0$, since there is insufficient recruitment in the absence of NCAs and NCAs stimulate recruitment. This NCA policy achieves efficiency provided the environment is such that $Z=Z(1,-\infty)>Z^{S P}$, which necessarily holds. ${ }^{26}$ As the minimum wage increases and becomes binding, optimal NCA policy becomes increasing in the minimum wage (i.e. $\gamma^{*}\left(w_{\min }\right)>0$ with $\frac{\partial \gamma^{*}\left(w_{\min }\right)}{\partial w_{\min }}>0$ for $\left.w_{\min }>w_{\min }^{L}\right)$ and achieves efficiency until NCA policy reaches $\gamma^{*}\left(w_{\min }\right)=1$. The only difference from the first case is that the threshold $w_{\text {min }}^{L}$ now coincides with the point at which the minimum wage becomes binding, $w_{\min }=R$.

We summarize this characterization of the interaction between optimal NCA policy and the minimum wage with the following proposition.

Proposition 6 (Necessity and sufficiency of two instruments to guarantee efficiency). Neither NCA policy nor minimum wage policy alone is sufficient to ensure that the constrained-efficient allocation

\footnotetext{
${ }^{25}$ See Proposition B1 in Appendix B for a formal proof of this claim.

${ }^{26}$ As $\gamma \rightarrow 1$ with no minimum wage, job-to-job transitions are entirely choked off, so we return to a Diamond-paradox economy in which all firms choose $w=R=b$. Inspection of the efficiency condition in (32) reveals that this situation necessarily entails excess recruitment.
} 
Figure 1: Optimal NCA policy

(a) NCA ban can be optimal

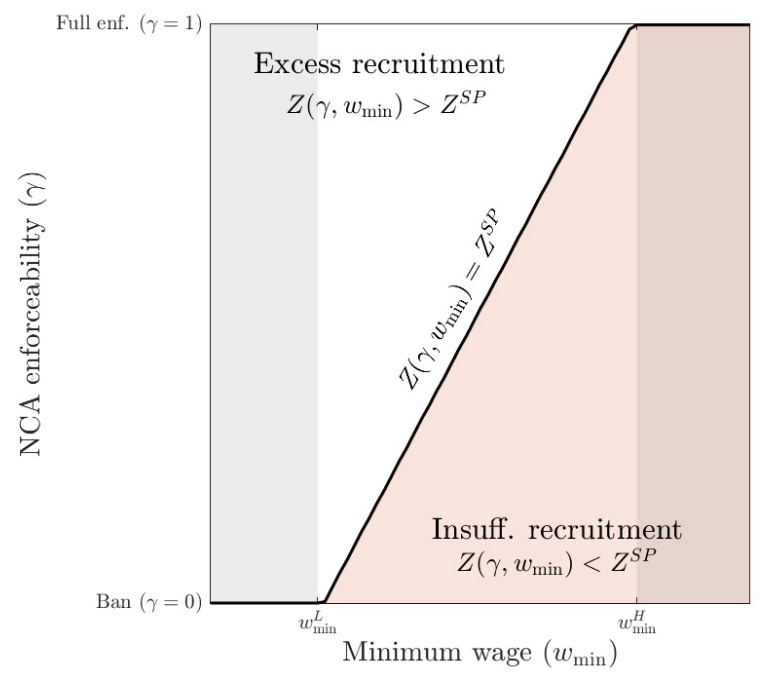

(b) NCA ban never optimal

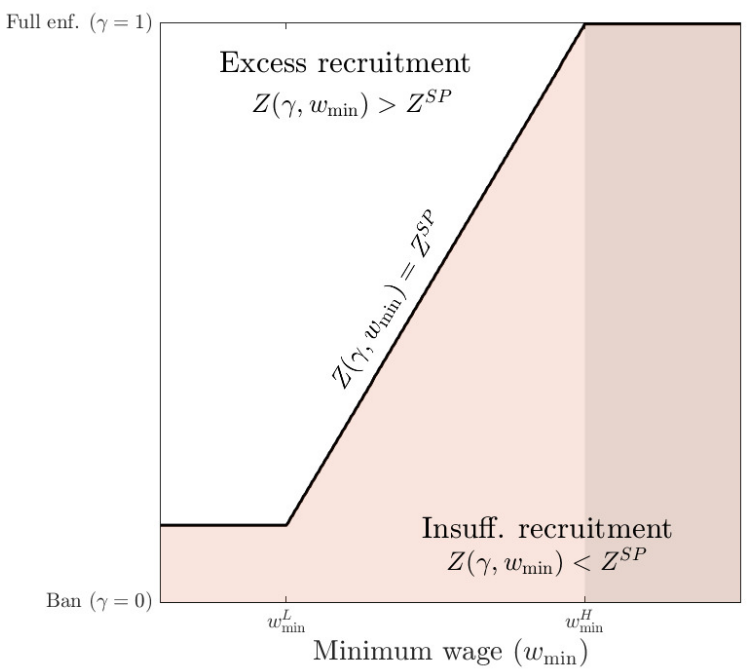

Panel 2a corresponds to an economy with a low value of $\epsilon_{Z}^{\lambda^{n}}$ (and thus strong congestion externalities) and panel $2 \mathrm{~b}$ corresponds to an economy with a high value of $\epsilon_{Z}^{\lambda^{n}}$ (and thus weak congestion externalities). The heavy line represents the optimal value of NCA enforceability, $\gamma^{*}$, for a given value of the minimum wage. The area above (below) this curve represents the region in which there is excessive (insufficient) recruitment in the decentralized equilibrium. The dark shaded areas to the left and right represent the values of the minimum wage for which NCA policy alone cannot implement the social optimum $Z=Z^{S P}$.

can always be achieved. With both policy instruments, however, the efficient allocation can always be achieved.

\section{Proof. See Appendix C.}

The intuition for this result follows directly from the preceding characterization. More generally, the existence of matching frictions unrelated to NCAs implies that both NCA policy and minimum wage policy are constrained instruments: There is a limit on the extent to which weakening NCAs can alleviate matching frictions and thus deter recruitment; likewise, because the minimum wage ceases to have an effect below the reservation wage, there is a limit on the extent to which reducing the minimum wage can stimulate recruitment. Hence, in the presence of excessive recruitment, while NCA policy can sometimes be used to achieve efficiency, it cannot always do so. Vice versa, in the presence of insufficient recruitment, while minimum wage policy can sometimes be used to achieve efficiency, it cannot always do so. Critically, however, because the two instruments are constrained in opposite directions, the combination of the two is always sufficient to ensure that the efficient allocation can always be achieved: If there is excess recruitment, for any level of NCA enforcement, a sufficiently high minimum wage can always achieve efficiency, whereas if there is 
insufficient recruitment, a sufficiently low minimum wage and sufficiently restrictive NCA policy can always achieve efficiency.

\section{Are NCAs Inefficient in Low-wage Labor Markets?}

What does the preceding characterization of optimal NCA policy imply for real-world labor markets? In particular, does prohibiting NCAs in low-wage labor markets increase welfare? The answer to this question generally depends on the calibration of the model and in particular on the level of the minimum wage. Nevertheless, in this final section we demonstrate that it is possible to derive a sufficient statistic for the inefficiency of NCAs that eliminates much of this parameter dependence. We show that this condition is likely to be satisfied in low-wage labor markets, both with and without binding minimum wages, implying that weakening NCAs will increase labor market efficiency. Then we consider a fully calibrated version of the model with parameters chosen to match Oregon in 2006, prior to its NCA ban. We show that Oregon's NCA ban was modestly efficiency-enhancing, but insufficient to achieve the constrained-efficient allocation. Doing so would have required a large increase in the minimum wage, the welfare gains from which would have been substantial.

\subsection{A sufficient statistic for weakening NCAs}

Suppose a policy maker is considering weakening NCAs. Would such a reform be welfare-enhancing? The following proposition shows that there is a simple answer to this question in the form of a threshold value for the level of employment (which, in our model, is effectively the employmentto-population ratio) above which NCAs are necessarily inefficiently restrictive. ${ }^{27}$ In the case of a non-binding minimum wage, the threshold depends only on the elasticity of the match rate for non-employed workers - a moment that has been estimated in the literature. In the case of a binding minimum wage, the threshold depends on two additional moments-labor's share of income and the mean-min wage ratio - moments that we can observe directly in the data.

Proposition 7 (Sufficient conditions for inefficiency of NCAs). There is excess recruitment in the decentralized equilibrium and weakening NCAs is efficiency enhancing if the following condition holds:

$$
e>2-\frac{1}{\epsilon_{Z}^{\lambda^{n}}}\left(\frac{p-\underline{w}}{p-R}\right) .
$$

In the case of a non-binding minimum wage, this condition reduces to:

$$
e>2-\frac{1}{\epsilon_{Z}^{\lambda^{n}}}
$$

\footnotetext{
${ }^{27}$ If we were to interpret non-employed workers as unemployed, the condition would yield a threshold for the unemployment rate below which recruitment is inefficiently high.
} 
In the case of a binding minimum wage, this condition is satisfied if:

$$
e>2-\frac{1}{\epsilon_{Z}^{\lambda^{n}}}\left(1-\eta^{L} / M m\right)
$$

where $\eta^{L} \equiv \frac{E[w]}{p}$ is labor's share of income and $M m \equiv \frac{E[w]}{w_{\min }}$ is the mean-min wage ratio.

Proof. See Appendix C.

Proposition 7 states that, for policy makers considering restricting the use of NCAs in low-wage labor markets, a sufficiently high employment rate ensures that this will be a social welfare-enhancing reform. The intuition flows from Proposition 1: A high level of employment is indicative of high - and possibly excessive - recruitment, in which case policy makers should weaken NCAs to deter recruitment. The dependence of the cutoff on $\epsilon_{Z}^{\lambda^{n}}$ reflects congestion externalities in firms' recruitment decisions as discussed in Section 5: Firms take aggregate recruitment intensity $Z$ as given and thus do not internalize the congestion caused by their individual recruitment decisions $z$ for other firms. The extent of this congestion is governed by the extent of diminishing returns in the aggregate matching function, which in turn is governed by $\epsilon_{Z}^{\lambda^{n}}$ : Higher values of $\epsilon_{Z}^{\lambda^{n}}$ imply weaker diminishing returns to aggregate recruitment, reduced congestion externalities, and thus a higher social tolerance for recruitment and a higher threshold above which we should attempt to deter recruitment by weakening NCAs. The dependence of the cutoff on labor's share and the mean-min wage ratio when the minimum wage is binding, in turn, reflects the fact that a high minimum wage implies a high share of income accruing to labor and thus relatively limited profits generated by new hires. A minimum wage thus effectively taxes recruitment, which mitigates the congestion externality described above and increases the threshold for the extent of recruitment that is socially excessive.

Figure 2 depicts the conditions in (34) (no minimum wage) and (35) (binding minimum wage) with $\epsilon_{Z}^{\lambda^{n}}$ on the horizontal axes and the level of employment on the vertical axes. The latter uses a value for $\eta^{L}$ of 0.47 , corresponding to the average cost share of labor in the Food Services and Drinking Places industry (NAICS 722) between 2010 and 2019, and a value for $M m$ of 1.27, corresponding to the mean-min wage ratio for prime-age workers with less than a high school degree, also in the Food Services and Drinking Places industry between 2010 and 2019. ${ }^{28}$

In both panels, the white area represents the region in which there is necessarily excessive recruitment and therefore, in light of Proposition 1, necessarily scope for weakening NCAs. The shaded red area represents the region where we cannot make a determination absent a more complete calibration of the model. Inspection of the figure quickly reveals that the value of $\epsilon_{Z}^{\lambda^{n}}$ is critical for assessing efficiency. If we interpret recruitment as vacancy creation, then this critical elasticity can be interpreted as the elasticity of matches from non-employment with respect to vacancies, an object

\footnotetext{
${ }^{28}$ Food Services and Drinking Places (NAICS 722) is the sector analyzed in studies of the effects of minimum wages on low-wage labor markets, such as Dube et al. (2016), and thus represents a natural benchmark given that the condition in (35) corresponds to the case of a binding minimum wage.
} 
Figure 2: Sufficient statistic for weakening NCAs

(a) Non-binding minimum wage

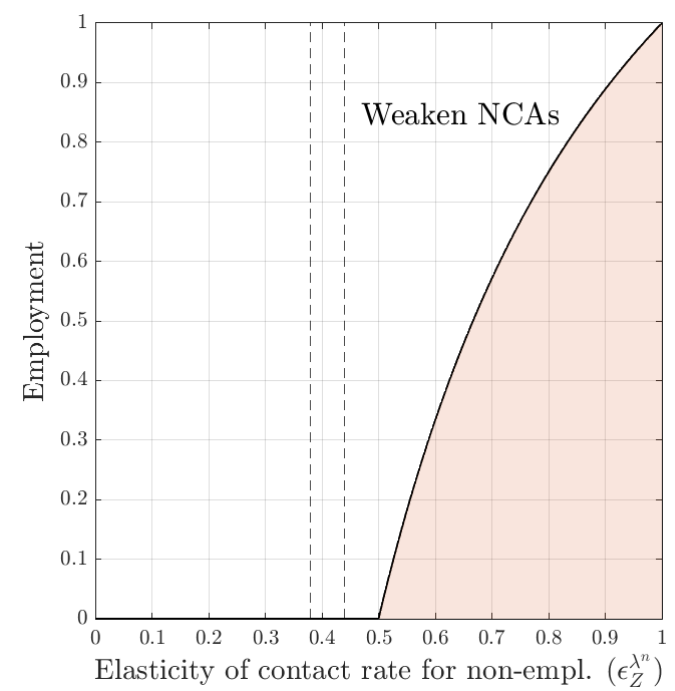

(b) Binding minimum wage

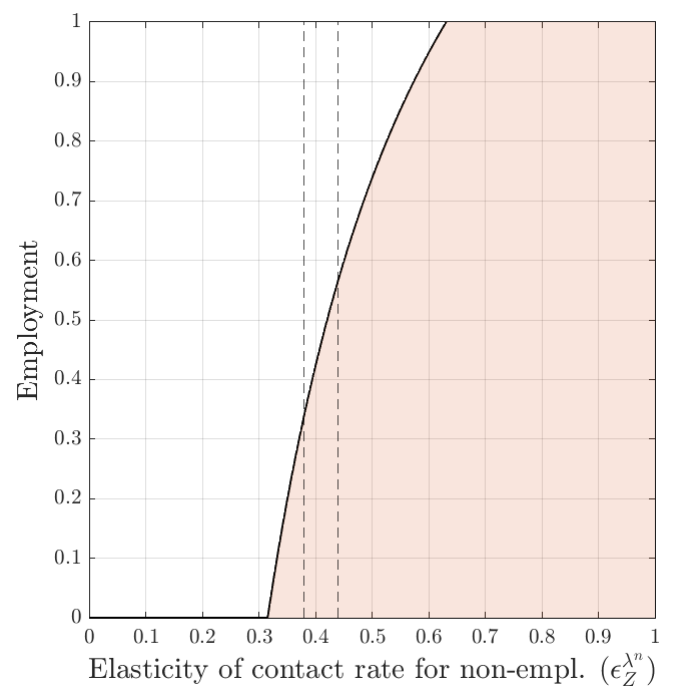

Panel 2a shows the case of a non-binding minimum wage. Panel $2 \mathrm{~b}$ shows the case of a binding minimum wage with $\eta^{L}=0.47$ and $M m=1.27$, both based on data for the Food Services and Drinking Places industry used in minimum wage studies (NAICS 722). The dashed lines corresponds to $\epsilon_{Z}^{\lambda^{n}}=\{0.38,0.44\}$ (based on Veracierto (2011) and Hall and Schulhofer-Wohl (2018), respectively). The white area represents the region in which there is excess recruitment, and hence in which weakening NCAs is necessarily efficiency-enhancing. In the red shaded area, the condition in Proposition 7 does not provide information on whether or not there is excess recruitment.

which has been studied in the literature. ${ }^{29}$ Recent work has found values close to 0.4: Specifically, Hall and Schulhofer-Wohl (2018) estimate a value of 0.44 based on all non-employed individuals who report wanting a job, and Veracierto (2011) estimates a value of 0.38 based on all non-employed workers. ${ }^{30}$ We indicate both values of $\epsilon_{Z}^{\lambda^{n}}$ with dashed vertical lines in the figure.

In the first panel, this elasticity is all that is needed to determine whether, for an observed level of employment, NCAs are inefficient. In particular, we see that at any level of employment NCAs should be weakened in order to deter excess recruitment. The second panel reflects the fact that a binding minimum wage will necessarily deter recruitment - just as weakening NCAs will deter recruitment - implying a higher cutoff for employment above which there is necessarily inefficiently high recruitment. Inspection of the second panel reveals that, based on values of $\eta^{L}$ and $M m$ from a frequently studied low-wage industry, weakening NCAs will be efficiency-enhancing in any low-wage labor market with employment in excess of between $55 \%$ and $60 \%$ (based on the more conservative

\footnotetext{
${ }^{29}$ Note that because we do not make a distinction between unemployed workers and workers who are out of the labor force, the relevant elasticity corresponds to matches from non-employment rather than matches from unemployment. We discuss the implications of re-interpreting non-employed workers in our model as unemployed below.

${ }^{30}$ Hall and Schulhofer-Wohl (2018) consider several other categorizations of non-employed workers that could also arguably correspond to non-employment in our model. For example, using workers who recently lost a permanent job yields a value of 0.49 .
} 
value of $\left.\epsilon_{Z}^{\lambda^{n}}\right)$.

What, if anything, does this imply about the efficiency of NCAs in observed low-wage labor markets? In the case of a non-binding minimum wage, we are able to immediately conclude that NCAs should be weakened, irrespective of the employment-to-population ratio. To assess the case of a binding minimum wage, we observe that the employment-to-population ratio for prime-age workers with less than a high school diploma ranged from $56 \%$ in 2010 to $62 \%$ in 2019 . The lowest of these values - $56 \%$ in the immediate aftermath of the Great Recession - lies close to the more-conservative threshold (i.e. the threshold based on the higher value of $\epsilon_{Z}^{\lambda^{n}}$ ) above which NCAs are inefficiently restrictive. This suggests that even with a binding minimum wage, weakening NCAs is likely to be welfare-enhancing.

It also bears noting that if we were to interpret non-employed workers in the model as unemployed (rather than non-employed), the vertical axis in Figure 2 would be interpreted as one minus the unemployment rate, in which case there would be no ambiguity about whether there is inefficiently high recruitment, particularly in the case of a binding minimum wage: In the data, the unemployment rate for the same group of workers described above ranges from 17\% (2010) to 6\% (2019) (corresponding to values on the vertical axis in Figure 2 of $83 \%$ and 94\%), while estimates of the elasticity of matches from unemployment with respect to vacancies are typically close to the values we use for the elasticity for non-employed workers (see, e.g., Petrongolo and Pissarides (2001)). Thus, in both panels, the case for weakening NCAs would be unequivocal if we were to re-interpret the model in this way.

\subsection{A quantitative assessment of optimal NCA policy}

Finally, we consider a full calibration of the model in order to quantify the welfare implications of NCAs and determine optimal NCA policy as a function of prevailing minimum wage levels. Furthermore, the calibrated model provides an illustration of the above-derived qualitative effects of NCA policy on key endogenous variables such as recruitment, the average wage and wage dispersion, and equilibrium employment.

\subsubsection{Functional forms}

A full calibration of the model requires that we take a stance on functional forms. We assume that the offer rates are iso-elastic and given by $\lambda^{n}(Z)=\mu^{n} Z_{Z}^{\epsilon_{Z}^{n}}$ and $\lambda^{e}(Z ; \gamma)=(1-\gamma) \mu^{e} Z^{\epsilon_{Z}^{\lambda^{e}}}$ where $\mu^{n}$ and $\mu^{e}$ govern the extent of underlying labor market frictions. Note that as $\gamma \rightarrow 1$ (NCAs are fully enforceable), there are no job-to-job transitions, while as $\gamma \rightarrow 0$ (NCAs are banned), only fundamental frictions restrict transitions. We also assume an iso-elastic form for the recruitment cost function $c(z)=\frac{c_{0}}{\epsilon_{Z}^{c}} Z^{\epsilon_{Z}^{c}}$.

\subsubsection{Parameter values}

Table 1 summarizes the calibration of the model. Our calibration focuses on low-wage labor markets, consistent with the analysis up to this point. Of particular note are our choices of the two policy 
parameters, $\gamma$ and $w_{\min }$. We calibrate both parameters using CPS data on prime-age (25-54) workers without a high school degree from Oregon in 2006, just before the state banned the use of NCAs for low-wage workers in 2008. ${ }^{31}$ Specifically, we calibrate $w_{\min }$ so that the model-implied ratio of the minimum wage to the median wage $\left(w_{\min } / w_{\text {median }}\right)$ is equal to the corresponding value in the data. $^{32}$ We calibrate the NCA enforceability parameter, $\gamma$, so that a full ban on NCAs in the model yields a $15 \%$ increase in the average rate of job-to-job transitions, $\chi$, the midpoint of the range of $12 \%$ to $18 \%$ identified by Lipsitz and Starr (2021) (see discussion in Section 2). ${ }^{33}$ We also calibrate the separation rate, $\delta$, to match the employment-to-population ratio of prime-age workers without a high school degree in Oregon in 2006 of $65 \%$. All remaining parameters are standard and based on data from workers with less than a high school degree when such data are available. See Appendix D for complete details of our calibration procedure.

Table 1: Calibration

\begin{tabular}{lllll}
\hline Concept & Param. & Value & Target & Reference \\
\hline Match output & $p$ & 1 & Normalization & \\
Value of nonempl. & $b$ & 0.73 & Opp. cost of empl. & Chodorow-Reich and Karabarbounis (2016) \\
Recr. cost (elast.) & $\epsilon_{z}^{c}$ & 2 & Quadratic & \\
Recr. cost (scale) & $c_{0}$ & 0.09 & $E\left[\frac{c^{\prime}(Z)}{w}\right]: \frac{0.05+0.14}{2}$ & Barron et al. (1997) \\
Contact eff. (E) & $\mu^{e}$ & 0.09 & $E E$ rate: 0.035 & Fallick and Fleischman (2001) \\
Contact eff. (N) & $\mu^{n}$ & 0.17 & $N E$ rate: 0.17 & Hall and Schulhofer-Wohl (2018) \\
Separation rate & $\delta$ & 0.09 & Emp/Pop: 0.65 & Authors' calculations \\
Contact elast. (E) & $\epsilon_{z}^{\lambda^{e}}$ & 0.26 & Match elast. (E) & Hall and Schulhofer-Wohl (2018) \\
Contact elast. (N) & $\epsilon_{z}^{\lambda^{n}}$ & 0.44 & Match elast. (N) & Hall and Schulhofer-Wohl (2018) \\
\hline NCA enforceability & $\gamma$ & 0.16 & $\frac{\chi_{B a n}-\chi_{N C A}=0.15}{\chi_{N C A}}$ & Lipsitz and Starr (2021) \\
Minimum wage & $w_{\min }$ & 0.67 & $w_{\min } / w_{\text {median }}$ & Authors' calculations \\
\hline
\end{tabular}

Notes: Parameter values chosen to match features of low-wage labor markets where data are available. Authors' calculations based on data from prime-age (25-54) workers with less than a high school degree in Oregon in 2006 (prior to the NCA ban for low-wage workers).

\subsubsection{Comparative statics revisited}

Figure 3 depicts how key endogenous variables in the model vary with the extent of NCA enforceability as measured by the parameter $\gamma$. Solid lines correspond to Oregon in 2006 prior to its NCA ban for low-wage workers, in which the minimum wage is calibrated so that the model-implied value of $w_{\min } / w_{\text {median }}$ matches the corresponding value in the data of 0.75 . Dashed lines correspond to a counterfactual high minimum wage economy in which the minimum wage is increased to match $w_{\min } / w_{\text {median }}=0.96$, the value from Washington, D.C. in $2019 .{ }^{34}$ The dashed vertical lines identify

\footnotetext{
${ }^{31}$ We use data from 2006 rather than 2007 to avoid any influence of the 2007-2008 financial crisis.

${ }^{32}$ State minimum wage data is from Ben Zipperer (https://github.com/benzipperer/historicalminwage/releases/tag/v1.2.0).

${ }^{33} \mathrm{An}$ alternative would have been to calibrate this parameter to match the elasticity of the average wage instead of the average rate of job-to-job transitions. We return to this possibility below.

${ }^{34}$ At the end of 2019 , D.C. had the highest value of $w_{\min } / w_{\text {median }}$ of 0.96 . We chose 2019 because it is the most recent year for which complete data are available and thus represents the high end of recently observed minimum
} 
our calibrated value of the NCA enforceability parameter $\gamma$ (see Table 1). ${ }^{35}$

Inspection of Figure 3 bears out the comparative statics results from Section 4: Weakening NCA enforceability reduces recruitment and employment, but increases job-to-job transitions, the average wage and wage dispersion. Furthermore, we observe that social welfare is monotonically decreasing in NCA enforceability. This is a stronger result than we obtained above in Sections 6.1, where our arguments effectively concerned local changes in NCAs. Here, we see that a full abolition of NCAs is efficiency enhancing, even in a high minimum wage economy.

Figure 3: Comparative statics
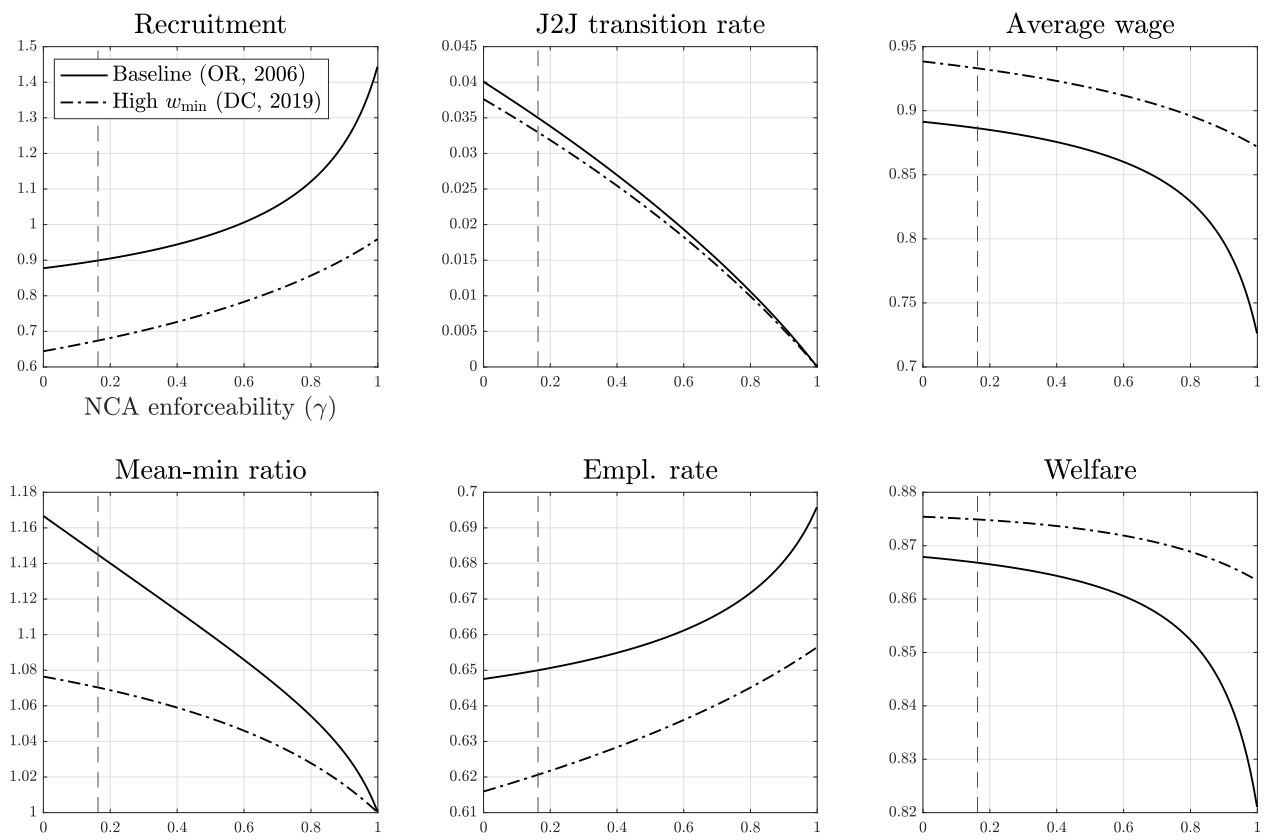

Figure 3 depicts how various endogenous variables respond to changes in the NCA enforceability parameter $\gamma$ based on the calibration described in the text. Solid lines correspond to the case of Oregon in 2006 prior to its NCA ban for lowwage workers, in which the minimum wage is calibrated to match the observed value of $w_{\min } / w_{\text {median }}=0.75$. Dashed lines correspond to a counterfactual scenario in which the minimum wage is increased to match $w_{\text {min }} / w_{\text {median }}=0.96$, the value from Washington, D.C. in 2019. Median wages are based on prime-age (25-54) full-time workers with less than a high school degree. Dashed vertical lines indicate the calibrated value of $\gamma$ for Oregon in 2006.

One advantage of a full calibration of the model is that it allows us to quantify the welfare gains from the elimination of NCAs for low-wage workers in Oregon in 2008. We find that Oregon's 2008 NCA ban had a small positive effect on efficiency, increasing social welfare by $0.13 \%$. As discussed above, these gains come from the fact that the observed level of enforcement of NCAs in Oregon prior to 2008 resulted in inefficiently high recruitment, so the NCA ban acted as a modest deterrent to recruitment. The model also predicts small negative employment effects associated with the ban - on the order of a one quarter of a percentage point reduction in the employment rate. The wage policy.

${ }^{35}$ Thus, the intersection of the solid lines with the dashed vertical lines indicate the values of endogenous variables in Oregon in 2006. 
relatively small effects of eliminating NCAs on welfare and employment are largely due to the fact that our calibration of $\gamma$ implies that NCA enforceability was relatively low to begin with - see the dashed vertical lines - and hence a full ban had only a limited effect on matching and thus recruitment and welfare.

We view these positive welfare effects as likely representing a lower bound for three reasons. First, our calibration procedure is conservative: Had we chosen $\gamma$ to match the observed increase in average wages associated with the Oregon NCA ban in Lipsitz and Starr (2021) (between 2\% and 3\%), the implied value of $\gamma$ would have been larger, resulting in a larger increase in welfare associated with a full elimination of NCAs. Second, our assumption that low-wage sectors of the economy do not feature significant productivity differentials across firms implies that there are no social benefits from workers climbing the job ladder. This implies that the welfare gains from relaxing mobility restrictions that we identify likely understate the gains that we would expect if job-to-job transitions were socially beneficial. ${ }^{36}$ Finally, as discussed above, we do not take a stance on whether workers who are not employed are unemployed or out of the labor force. If we had instead assumed that all such workers were unemployed, our calibration would have implied a higher level of employment, a higher level of recruitment, and thus larger welfare gains from deterring recruitment by banning NCAs.

\subsubsection{NCAs and the minimum wage}

That welfare is monotonically decreasing in NCA enforceability in both regimes in Figure 3 reflects fixed values of the minimum wage, calibrated to match the data. However, as we have discussed above, both relaxing NCAs and increasing the minimum wage necessarily reduce recruitment in the model, suggesting an important interaction between the two policies. In particular, the apparent substitutability of weakening NCAs and raising the minimum wage implies that at a sufficiently high level of the minimum wage, welfare will cease to be monotonically declining in the level of NCA enforceability as it is in Figure 3. To understand the nature of this relationship more clearly, Figure 4 plots social welfare as a function of $\gamma$ and $w_{\min }$ in our baseline calibration for the full range of values of $\gamma$ and for $w_{\min } \in[0.5, p]$.

The surface in Figure 4 features an efficient ridge, corresponding to the combinations of NCAs and the minimum wage that maximize social welfare. The contour of this ridge has a negative slope in $\left(\gamma, w_{\min }\right)$-space, reflecting the tradeoff between raising the minimum wage and weakening NCAs at the social optimum. We also observe in the figure that welfare losses can quickly become large - on the order of $5-6 \%$-in extreme regions of the parameter space corresponding to very high NCA enforceability with modest minimum wages or a very high minimum wage.

Returning to Figure 3 in light of this discussion, we see that in both the baseline and high minimum wage economies, eliminating NCAs is welfare-enhancing, yet not sufficient to entirely rid the economy of excess recruitment and restore efficiency. This is precisely the problem highlighted by the first part of Proposition 6: NCA policy alone cannot necessarily implement the social optimum. Nevertheless-

\footnotetext{
${ }^{36}$ Nevertheless, we view an assumption of no productivity differentials as a reasonable baseline given our focus on low-wage sectors of the economy.
} 
Figure 4: Policy interactions and social welfare

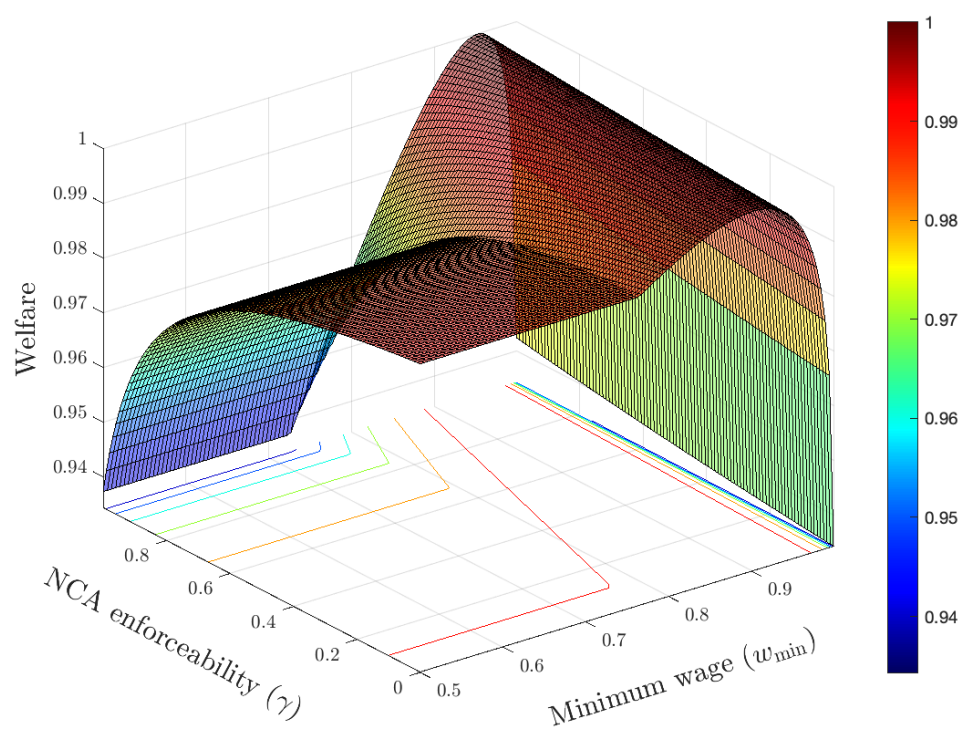

Figure 4 depicts how social welfare, $\Omega$, varies with NCA enforceability $(\gamma)$ and the minimum wage $\left(w_{\text {min }}\right)$. Higher values represent higher levels of social welfare. We normalize the welfare measure so that the social optimum corresponds to a value of 1 .

and consistent with the second part of Proposition 6-Figure 4 reveals that enabling policy makers to also raise the minimum wage can restore efficiency once NCAs have been eliminated. Put differently, NCAs and the minimum wage are substitutes locally - in the sense that from an interior efficient combination of $\gamma$ and $w_{\min }$, policy makers can generally reduce the minimum wage and weaken NCAs and remain at the efficient allocation-but complements globally, in the sense that only with both policies in hand can policy makers ensure that the social optimum is achieved.

In fact, the calibrated model allows us to identify the level of the minimum wage that would have been optimal in Oregon following its 2008 NCA ban, and also to compute the welfare gains associated with such a hypothetical change. To this end, Figure 5 depicts the percent change in the social welfare criterion $\Omega$ associated with (i) the elimination of NCAs for low-wage workers in Oregon in 2008 (as discussed above), and (ii) a counterfactual scenario in which, after eliminating NCAs, policy makers also increased the minimum wage to its socially optimal level. The vertical arrow indicates the welfare gain from the (implemented) elimination of NCAs, and the horizontal arrow indicates the welfare gain from a (hypothetical) increase in the minimum wage to its welfare-maximizing level.

The figure highlights that, following the NCA ban, there was considerable scope for policy makers to increase the minimum wage: Based on the calibrated model, the optimal minimum wage following the NCA ban would have been $\$ 10.03$ per hour, more than $30 \%$ higher than the actual minimum wage in 2006 of $\$ 7.50$ per hour (both in 2006 current dollars). Such an increase in the minimum wage would have increased social welfare by $0.94 \%$, implying that eliminating NCAs and implementing 
Figure 5: Efficiency gains and optimal policy

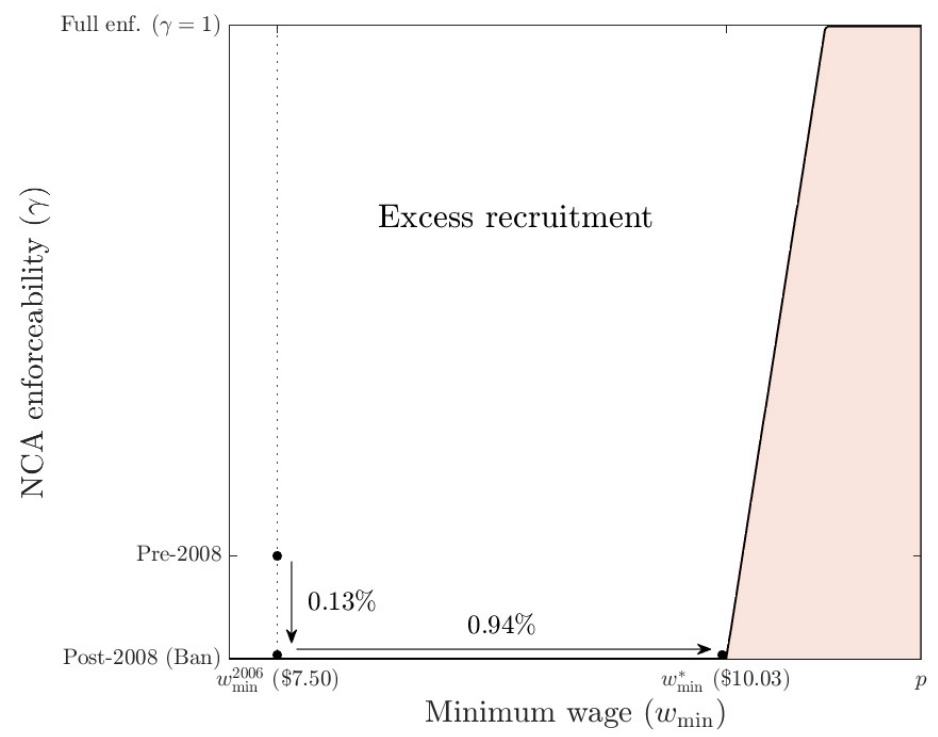

Figure 5 depicts the welfare gains $(\% \Delta \Omega)$ from (i) Oregon's NCA ban (vertical arrow) and (ii) a hypothetical increase in the minimum wage from its actual level in 2006 (\$7.50) to its welfare-maximizing level (\$10.03) (horizontal arrow). Note that both the actual and welfare-maximizing values of the minimum wage are measured in 2006 current dollars.

the optimal minimum wage together would have increased social welfare by roughly one $1 \%$.

\section{Conclusion}

We study the efficiency of NCAs in low-wage labor markets. In the context of a model of wage posting with endogenous recruitment that is consistent with the empirical effects of NCAs, we show that NCAs necessarily stimulate recruitment and thus job creation, giving rise to an efficiency rationale for NCA enforcement. Nevertheless, through a sufficient statistic analysis and a calibration of the model, we argue that NCAs are excessively restrictive and that enforceability should be weakened on efficiency grounds. Furthermore, we show that implementing the socially efficient allocation requires increasing minimum wages beyond currently-observed levels. More broadly, our analysis shows that the optimal level of NCA enforceability depends on the level of the minimum wage. Thus, it is important to jointly consider policy choices about minimum wages and NCAs rather than discussing them separately. 


\section{References}

Barron, J., M. Berger, and D. Black (1997). On the Job Training. Upjohn Institute.

Bishara, N. (2011). Fifty ways to leave your employer: Relative enforcement of noncompete agreements, trends, and implications for employee mobility policy. University of Pennsylvania Journal of Business Law.

Bishara, N., K. Martin, and R. Thomas (2015). An empirical analysis of noncompetition clauses and other restrictive postemployment covenants. Vanderbilt Law Review.

Bishara, N. and E. Starr (2016). The incomplete noncompete picture. Lewis and Clark Law Review.

Brenzel, H., H. Gartner, and C. Schnabel (2014). Wage bargaining or wage posting? evidence from the employers' side. Labour Economics.

Burdett, K. and D. Mortensen (1998). Wage differentials, employer size, and unemployment. International Economic Review.

Cahuc, P., S. Carcillo, and A. Zylberberg (2014). Labor Economics. MIT Press.

Chodorow-Reich, G. and L. Karabarbounis (2016). The cyclicality of the opportunity cost of employment. Journal of Political Economy.

Coles, M. and D. Mortensen (2016). Equilibrium labor turnover, firm growth, and unemployment. Econoemtrica.

Colvin, A. and H. Shierholz (2019). Noncompete agreements. Technical report, Economic Policy Institute.

Doniger, C. and D. Toohey (2021). On the efficiency of progressive social insurance and a novel test for posted wages. Unpublished manuscript.

Dube, A., T. W. Lester, and M. Reich (2016). Minimum wage shocks, employment flows, and labor market frictions. Journal of Labor Economics.

Faberman, R. J., A. I. Mueller, A. Şahin, and G. Topa (2017, August). Job Search Behavior among the Employed and Non-Employed. NBER Working Papers 23731, National Bureau of Economic Research, Inc.

Fallick, B. and C. Fleischman (2001). The importance of employer-to-employer flows in the u.s. labor market. Federal Reserve Working Paper.

Galle, W. and C. Koen (2001). Reducing post-termination disputes: A national survey of contract clauses used in employment contracts. Journal of Individual Employment Rights.

Garmaise, M. (2011). Ties that truly bind: Noncompetition agreements, executive compensation, and firm investment. Journal of Law, Economics, and Organization. 
Hall, R. and A. Krueger (2012). Evidence on the incidence of wage posting, wage bargaining, and on-the-job search. American Economic Journal: Macroeconomics.

Hall, R. and S. Schulhofer-Wohl (2018). Measuring job-finding rates and matching efficiency with heterogeneous job-seekers. American Economic Journal: Macroeconomics 10(1).

Hornstein, A., P. Krusell, and G. Violante (2011). Frictional wage dispersion in search models: A quantitative assessment. American Economic Review.

Hosios, A. J. (1990). On The Efficiency of Matching and Related Models of Search and Unemployment. Review of Economic Studies 57(2), 279-298.

Kaplan, S. and P. Stromberg (2003). Financial contracting theory meets the real world: An empirical analysis of venture capital contracts. Review of Economic Studies.

Krueger, A. and O. Ashenfelter (2018). Theory and evidence on employer collusion in the franchise sector. NBER Working Paper.

Lavetti, K., C. Simon, and W. White (2019). The impacts of restricting mobility of skilled service workers: Evidence from physicians. Journal of Human Resources.

Lipsitz, M. and E. Starr (2021). Low-wage workers and the enforceability of non-compete agreements. Management Science.

Manning, A. (2003). Monopsony in Motion. Princeton University Press.

Manning, A. (2006). A generalised model of monopsony. Economic Journal 116(508).

Manning, A. (2011). Imperfect competition in the labor market. Handbook of Labor Economics 4 b.

Marx, M. (2011). The firm strikes back: Non-compete agreements and the mobility of technical professionals. American Sociological Review.

Nagypal, E. (2008). Worker reallocation over the business cycle: The importance of employer-toemployer transitions. Manuscript, Northwestern University.

Petrongolo, B. and C. Pissarides (2001). Looking into the black box: A survey of the matching function. Journal of Economic Literature.

Schwab, S. and R. Thomas (2006). An empirical analysis of ceo employment contracts: What do top executives bargain for? Washington and Lee Law Review.

Starr, E., J. Prescott, and N. Bishara (2021). Noncompetes in the u.s. labor force. The Journal of Law and Economics.

Veracierto, M. (2011). Worker flows and matching efficiency. Economic Perspectives 35(4).

Young, S. (2021). Noncompete clauses, job mobility, and job quality: Evidence from a low-earning noncompete ban in austria. mimeo. 


\section{Appendices}

\section{A Model Details}

\section{A.1 Derivation of offer distribution}

In this appendix we derive equations (19), (21) and (22) in Section 3 of the text. ${ }^{37}$ The derivation follows Cahuc et al. (2014).

To derive (19), we begin with two relationships implied by the model. The first is equation (18) from the body of the text (which we repeat below). The second follows from the fact that the mass of employees receiving wage $w$ can be written either as $l(w) H^{\prime}(w)$ or as $e G^{\prime}(w)$. Thus, we have

$$
\begin{gathered}
G(w)=\frac{H(w)}{1+\lambda^{e} / \delta(1-H(w))} \\
G^{\prime}(w) e=l(w) H^{\prime}(w) .
\end{gathered}
$$

Differentiating (A.1) with respect to $w$ yields

$$
G^{\prime}(w)=\frac{H^{\prime}(w)\left[1+\left(\lambda^{e} / \delta\right) G(w)\right]}{1+\left(\lambda^{e} / \delta\right)(1-H(w))}
$$

or, equivalently,

$$
\frac{G^{\prime}(w)}{1+\left(\lambda^{e} / \delta\right) G(w)}=\frac{H^{\prime}(w)}{1+\left(\lambda^{e} / \delta\right)(1-H(w))} .
$$

Substituting (A.3) into (A.2), we obtain

$$
\left[1+\lambda^{e} / \delta G(w)\right] e=l(w)\left[1+\lambda^{e} / \delta(1-H(w))\right]
$$

Taking logs, differentiating with respect to $w$ and rearranging gives

$$
\lambda^{e} / \delta\left(\frac{G^{\prime}(w)}{1+\lambda^{e} / \delta G(w)}\right)=\frac{l^{\prime}(w)}{l(w)}-\frac{\lambda^{e} / \delta H^{\prime}(w)}{1+\lambda^{e} / \delta(1-H(w))} .
$$

Substituting (A.4) into the left-hand side of (A.6) and rearranging, we obtain

$$
\frac{l^{\prime}(w)}{l(w)}=\frac{2\left(\lambda^{e} / \delta\right) H^{\prime}(w)}{1+\left(\lambda^{e} / \delta\right)(1-H(w))} .
$$

Next, we can use (A.7) together with the first-order condition for $w$ in the body of the text, (14), to obtain a first-order differential equation in $H(w)$ :

$$
2(p-w) H^{\prime}(w)+H(w)=\frac{1+\lambda^{e} / \delta}{\lambda^{e} / \delta} .
$$

\footnotetext{
${ }^{37}$ For ease of notation, we suppress dependence of endogenous variables on $Z$ and $\gamma$ throughout.
} 
The general solution to this differential equation is

$$
H(w)=d(p-w)^{\frac{1}{2}}+\frac{1+\lambda^{e} / \delta}{\lambda^{e} / \delta}
$$

where $d$ is a constant. The value of $d$ is obtained by observing that no firm will offer a wage less that $w=\underline{w}$, so $H(\underline{w})=0$. Using this in (A.9), we obtain

$$
d=-\frac{1+\lambda^{e} / \delta}{\lambda^{e} / \delta}(p-\underline{w})^{-\frac{1}{2}}
$$

which can be substituted back into (A.9) to obtain the unique solution for $H(w)$ in (19) in the body of the text, namely

$$
H(w)=\frac{1+\lambda^{e} / \delta}{\lambda^{e} / \delta}\left[1-\left(\frac{p-w}{p-\underline{w}}\right)^{\frac{1}{2}}\right] .
$$

The upper bound of the wage distribution in equation (22) in the body of the text is the wage that yields $H(w)=1$ in (A.11):

$$
\bar{w}=p-(p-\underline{w})\left(\frac{1}{1+\lambda^{e} / \delta}\right)^{2} .
$$

Finally, the reservation wage in equation (21) in the body of the text is obtained by substituting the solution for $H(w)$ in (A.11) into (5) and solving for $R$ (given that $\underline{w}=R$ ):

$$
R=\frac{b\left(\delta+\lambda^{e}\right)^{2}+p\left(\lambda^{n}-\lambda^{e}\right) \lambda^{e}}{\left(\delta+\lambda^{e}\right)^{2}+\left(\lambda^{n}-\lambda^{e}\right) \lambda^{e}}
$$

\section{A.2 Equilibrium recruitment $(Z)$}

We next consider some features of the implicit function defining equilibrium recruitment in (23) that we will use throughout the appendix. First, note that we can rewrite (23) as

$$
c^{\prime}(Z)=\Pi\left(\lambda^{e}(Z ; \gamma), \lambda^{n}(Z)\right) \cdot r_{z}^{n}(Z)
$$

where the left-hand side is the marginal cost of recruitment and the right-hand side is the product of the expected lifetime profits generated by a new hire and the inflow of new hires associated with extra recruitment effort, i.e.

$$
\begin{aligned}
& \Pi\left(\lambda^{e}(Z ; \gamma), \lambda^{n}(Z)\right)=\left\{\begin{array}{ll}
\frac{(p-b)\left(\delta+\lambda^{e}(Z ; \gamma)\right)}{\left(\delta+\lambda^{e}(Z ; \gamma)\right)^{2}+\lambda^{e}(Z ; \gamma)\left(\lambda^{n}(Z)-\lambda^{e}(Z ; \gamma)\right)} & \text { if } w_{\min } \leq R \\
\frac{p-w}{\delta+\lambda^{e}(Z ; \gamma)} & \text { if } w_{\min }>R
\end{array}>0\right. \\
& r_{z}^{n}(Z)=\frac{\delta \lambda^{n}(Z)}{Z\left(\delta+\lambda^{n}(Z)\right)}>0
\end{aligned}
$$

where the inequalities hold because, as we show in the body of the text, it must be that $Z>0$. 
Using the expressions in (A.15) and (A.16), it is straightforward to verify the following:

$$
\begin{aligned}
& r_{z Z}^{n}=\frac{\delta^{2} \lambda^{n}(Z)\left(\epsilon_{Z}^{\lambda^{n}}-1\right)-\delta\left(\lambda^{n}(Z)\right)^{2}}{Z^{2}\left(\delta+\lambda^{n}(Z)\right)^{2}}<0 \\
& \Pi_{\lambda^{e}}= \begin{cases}-\frac{(p-b) \delta\left(\delta+\lambda^{n}(Z)\right)}{\left(\delta^{2}+2 \delta \lambda^{e}(Z ; \gamma)+\lambda^{e}(Z ; \gamma) \lambda^{n}(Z)\right)^{2}} & \text { if } w_{\min } \leq R \\
-\frac{1}{\left(\delta+\lambda^{e}(Z ; \gamma)\right)^{2}} & \text { if } w_{\min }>R\end{cases} \\
& \Pi_{\lambda^{n}}= \begin{cases}-\frac{(p-b) \lambda^{e}(Z ; \gamma)\left(\delta+\lambda^{e}(Z ; \gamma)\right)}{\left(\delta^{2}+2 \delta \lambda^{e}(Z ; \gamma)+\lambda^{e}(Z ; \gamma) \lambda^{n}(Z)\right)^{2}} & \text { if } w_{\min } \leq R \\
0 & \text { if } w_{\min }>R\end{cases}
\end{aligned}
$$

where $r_{z Z}^{n}$ is the derivative of (A.16) with respect to $Z$ (and the sign of the derivative follows from concavity of $\lambda^{n}$ in $Z$ ), $\Pi_{\lambda^{e}}$ is the derivative of (A.15) with respect to its first argument, and $\Pi_{\lambda^{n}}$ is the derivative of (A.15) with respect to its second argument. We will use (A.14)-(A.16) and the inequalities in (A.17)-(A.19) extensively below.

\section{A.3 Uniqueness and existence of $Z$}

Uniqueness: Convexity of $c(\cdot)$ implies that the left-hand side of (A.14) is increasing. Differentiating the right-hand side with respect to $Z$, we obtain

$$
\Pi \cdot r_{z Z}^{n}+r_{z}^{n} \cdot\left(\Pi_{\lambda^{e}} \lambda_{Z}^{e}+\Pi_{\lambda^{n}} \lambda_{Z}^{n}\right)<0
$$

where the inequality follows from the inequalities in (A.17)-(A.19) together with the fact that offer rates are increasing in recruitment $\left(\lambda_{Z}^{e}>0\right.$ and $\left.\lambda_{Z}^{n}>0\right)$ and the fact that the components of the marginal benefit of recruitment in (A.15) and (A.16) are positive ( $\Pi>0$ and $\left.r_{z}^{n}>0\right)$. Thus, there can be at most one value of $Z$ that satisfies (A.14). Note that this argument does not depend on strict convexity of $c(\cdot)$, a fact that will be useful when we consider a model with free entry instead of recruitment in Appendix E.

Existence: To see that there exists a value of $Z$ satisfying (A.14), we consider the limits of (A.14) as $Z$ approaches zero and positive infinity. As $Z$ approaches zero, we have

$$
\lim _{Z \rightarrow 0} c^{\prime}(Z)=0<\left(\frac{p-\max \left\{b, w_{\min }\right\}}{\delta}\right) \cdot \lambda_{Z}^{n}(0)=\lim _{Z \rightarrow 0}\left\{\Pi\left(\lambda^{e}(Z ; \gamma), \lambda^{n}(Z)\right) \cdot r_{z}^{n}(Z)\right\}
$$

where the inequality follows from $p>\max \left\{b, w_{\min }\right\}$ and the assumption that $\lambda^{n}$ is strictly increasing so that $\lambda_{Z}^{n}(0)>0$. Thus, the right-hand side of (A.14) strictly exceeds the left-hand side as $Z$ approaches zero. To evaluate the limit as $Z$ approaches positive infinity, write

$$
\lim _{Z \rightarrow \infty}\left\{\Pi\left(\lambda^{e}(Z ; \gamma), \lambda^{n}(Z)\right) \cdot r_{z}^{n}(Z)\right\}=\lim _{Z \rightarrow \infty}\left\{\Pi\left(\lambda^{e}(Z ; \gamma), \lambda^{n}(Z)\right)\right\} \cdot \lim _{Z \rightarrow \infty}\left\{r_{z}^{n}(Z)\right\}
$$


Now, observe that, using (A.16), we have

$$
\lim _{Z \rightarrow \infty}\left\{r_{z}^{n}(Z)\right\}=\lim _{Z \rightarrow \infty}\left\{\frac{\delta \lambda^{n}(Z)}{Z\left(\delta+\lambda^{n}(Z)\right)}\right\}=\lim _{Z \rightarrow \infty}\left\{\frac{\delta \lambda_{Z}^{n}(Z)}{\delta+\lambda^{n}(Z)+Z \lambda_{Z}^{n}(Z)}\right\}=0
$$

where the second equality follows from l'Hopital's rule. Furthermore, observe that if the minimum wage is binding, (A.15) immediately implies $\lim _{Z \rightarrow \infty}\left\{\Pi\left(\lambda^{e}(Z ; \gamma), \lambda^{n}(Z)\right)\right\}=0$, whereas if the minimum wage is non-binding, we can use (A.15) to write

$$
\begin{aligned}
\lim _{Z \rightarrow \infty}\left\{\Pi\left(\lambda^{e}(Z ; \gamma), \lambda^{n}(Z)\right)\right\} & =\lim _{Z \rightarrow \infty}\left\{\frac{(p-b)\left(\delta+\lambda^{e}(Z ; \gamma)\right)}{\left(\delta+\lambda^{e}(Z ; \gamma)\right)^{2}+\lambda^{e}(Z ; \gamma)\left(\lambda^{n}(Z)-\lambda^{e}(Z ; \gamma)\right)}\right\} \\
& =\lim _{Z \rightarrow \infty}\left\{\frac{(p-b) \lambda_{Z}^{e}(Z ; \gamma)}{\lambda_{Z}^{e}(Z)\left(2 \delta+\lambda^{n}(Z)\right)+\lambda_{Z}^{n}(Z) \lambda^{e}(Z ; \gamma)}\right\} \\
& =0
\end{aligned}
$$

where again the second equality follows from l'Hopital's rule. Using these results in (A.22), we have

$$
\lim _{Z \rightarrow \infty}\left\{\Pi\left(\lambda^{e}(Z ; \gamma), \lambda^{n}(Z)\right) \cdot r_{z}^{n}(Z)\right\}=0<\lim _{Z \rightarrow \infty} c^{\prime}(Z)
$$

Thus, the left-hand side of (A.14) strictly exceeds the right-hand side as $Z$ approaches positive infinity. It follows from the preceding that there must exist a value of $Z$ that solves (A.14).

Because we have shown that there is at most one solution to (A.14), and that a solution must exist, it follows that there is a unique equilibrium level of recruitment $Z$ as claimed in the text. 


\section{B Comparative Statics}

In this appendix we provide details of the proofs and derivations found in Section 4 of the text.

\section{B.1 Recruitment and offer rates (Propositions 1 and 2)}

We first provide proofs for Propositions 1 and 2, which characterize the effects of NCAs on recruitment $(Z)$ and the offer rate of employed workers $\left(\lambda^{e}\right) .{ }^{38}$

\section{B.1.1 Recruitment (Proposition 1)}

Proof. Because $Z$ is continuous in $\gamma$, to establish that recruitment is increasing in NCA enforceability, it is sufficient to establish that $d Z / d \gamma>0$ for the two relevant cases: a non-binding minimum wage and a binding minimum wage. ${ }^{39}$ We consider both cases together, using (A.14), (A.15) and (A.16). Differentiating (A.14) with respect to $\gamma$, taking account of the implicit dependence of $Z$ on $\gamma$, and solving for $d Z / d \gamma$, gives

$$
\frac{d Z}{d \gamma}=\frac{r_{z}^{n} \Pi_{\lambda^{e}} \lambda_{\gamma}^{e}}{c^{\prime \prime}(Z)-r_{z}^{n}\left(\Pi_{\lambda^{e}} \lambda_{Z}^{e}+\Pi_{\lambda^{n}} \lambda_{Z}^{n}\right)-\Pi r_{z Z}^{n}}>0
$$

where the sign follows from (i) convexity of $c(\cdot)$ which implies $c^{\prime \prime}(Z)>0$, (ii) (A.15)-(A.19) from which we know $\Pi>0, r_{z}^{n}>0, r_{z Z}^{n}<0, \Pi_{\lambda^{e}}<0$ and $\Pi_{\lambda^{n}} \leq 0$, and (iii) the assumptions in Section 3.1.1 which imply $\lambda_{\gamma}^{e}<0, \lambda_{Z}^{e}>0$, and $\lambda_{Z}^{n}>0$ (where $\lambda_{\gamma}^{e} \equiv \frac{\partial \lambda^{e}}{\partial \gamma}$ is the partial derivative of $\lambda^{e}(Z ; \gamma$ ) with respect to $\gamma$ and thus only reflects the direct effect of $\gamma$ on $\lambda^{e}$ ). Note that this result holds for both a non-binding and for a binding minimum wage.

\section{B.1.2 Offer rate (Proposition 2)}

Proof. It is again sufficient to establish that $d \lambda^{e} / d \gamma<0$ for the two relevant cases: a non-binding minimum wage and a binding minimum wage. Once again differentiating (A.14) with respect to $\gamma$, this time in such a way that allows us to solve for $d \lambda^{e} / d \gamma$ (that is, the total effect of $\gamma$ on $\lambda^{e}(Z ; \gamma)$, taking account of the direct effect and the indirect effect through which $\gamma$ affects $Z$ ), we obtain

$$
\frac{d \lambda^{e}}{d \gamma}=\frac{d Z}{d \gamma}\left(\frac{c^{\prime \prime}(Z)-\Pi r_{z Z}^{n}-r_{z}^{n} \Pi_{\lambda^{n}} \lambda_{Z}^{n}}{r_{z}^{n} \Pi_{\lambda^{e}}}\right)<0
$$

where the sign follows from from (i) convexity of $c(\cdot)$ which implies $c^{\prime \prime}(Z)>0$, (ii) (A.15)-(A.19) from which we know $\Pi>0, r_{z}^{n}>0, r_{z Z}^{n}<0, \Pi_{\lambda^{e}}<0$ and $\Pi_{\lambda^{n}} \leq 0$, (iii) the assumptions in Section 3.1.1 which imply $\lambda_{\gamma}^{e}<0, \lambda_{Z}^{e}>0$, and $\lambda_{Z}^{n}>0$, and (iv) the fact that $d Z / d \gamma>0$ (as proved above). Note once again that this result holds for both a non-binding and for a binding minimum wage.

\footnotetext{
${ }^{38}$ Both proofs make extensive use of the inequalities in (A.15)-(A.19).

${ }^{39}$ Changes in NCA enforceability, $\gamma$, affect workers' reservation wages, $R$, and may thus affect whether or not the minimum wage is binding. This implies that there is a kink in the relationship between $Z$ and $\gamma$ at $w_{\text {min }}=R$, but no discontinuity.
} 


\section{B.2 Mobility and wages (Proposition 4)}

We next provide the proof for Proposition 4, which characterizes the effects of NCAs on the average rate of job-to-job transitions $(\chi)$, the average wage $(E[w])$, and wage dispersion as measured by the mean-min ratio $(\mathrm{Mm})$.

Proof. We consider the three variables in turn.

Job-to-job transitions. Temporarily suppressing dependence of endogenous variables on $Z$ and $\gamma$ for ease of notation, the average job-to-job transition rate in the model is given by

$$
\chi \equiv \int_{\underline{w}}^{\bar{w}} \lambda^{e}(1-H(w)) d G(w) .
$$

Following the derivation in Hornstein et al. (2011), which in turn is based on Nagypal (2008), integrating by parts yields

$$
\begin{aligned}
\chi & =\lambda^{e}-\lambda^{e} \int_{\underline{w}}^{\bar{w}} H(w) d G(w) \\
& =\lambda^{e}-\lambda^{e}[H(w) G(w)]_{\underline{w}}^{\bar{w}}+\lambda^{e} \int_{\underline{w}}^{\bar{w}} G(w) d H(w) \\
& =\lambda^{e} \int_{\underline{w}}^{\bar{w}} G(w) d H(w) \\
& =\lambda^{e} \delta \int_{\underline{w}}^{\bar{w}} \frac{H(w)}{\delta+\lambda^{e}(1-H(w))} d H(w)
\end{aligned}
$$

where the last equation uses the solution for $G(w)$ in (18). Changing the variable of integration to $z=H(w)$, we have ${ }^{40}$

$$
\begin{aligned}
\int_{0}^{1} \frac{z}{\delta+\lambda^{e}(1-z)} d z & =-\frac{\lambda^{e}[z]_{0}^{1}+\left(\delta+\lambda^{e}\right)\left[\ln \left(\delta+\lambda^{e}(1-z)\right)\right]_{0}^{1}}{\left(\lambda^{e}\right)^{2}} \\
& =-\frac{\lambda^{e}+\left(\delta+\lambda^{e}\right)\left(\ln (\delta)-\ln \left(\delta+\lambda^{e}\right)\right)}{\left(\lambda^{e}\right)^{2}} \\
& =\frac{\left(\delta+\lambda^{e}\right) \ln \left(\frac{\delta+\lambda^{e}}{\delta}\right)}{\left(\lambda^{e}\right)^{2}}-\frac{1}{\lambda^{e}} .
\end{aligned}
$$

Thus, we obtain the equation in the text

$$
\chi=\delta\left[\frac{\delta+\lambda^{e}}{\lambda^{e}} \ln \left(\frac{\delta+\lambda^{e}}{\delta}\right)-1\right]
$$

\footnotetext{
${ }^{40}$ Because $H(\underline{w})=0$ and $H(\bar{w})=1$, the change of variables implies that the limits of integration become 0 and 1 . See Nagypal (2008) for details.
} 
Note that this expression for the average rate of job-to-job transitions does not depend on $\underline{w}$. Thus, the effect of NCAs on the average rate of job-to-job transitions does not depend on whether or not there is a binding minimum wage. Because (B.11) does not depend on $\lambda^{n}$, we can write $d \chi / d \gamma=\frac{d \chi}{d \lambda^{e}} \frac{d \lambda^{e}}{d \gamma}$, which implies that the sign of $d \chi / d \gamma$ will be the opposite of the sign of $d \chi / d \lambda^{e}$ since Proposition 2 tells us that $d \lambda^{e} / d \gamma<0$. Differentiating (B.11) with respect to $\lambda^{e}$ yields

$$
\begin{aligned}
\frac{d \chi}{d \lambda^{e}} & =\frac{\delta}{\lambda^{e}}\left[1-\ln \left(1+\frac{\lambda^{e}}{\delta}\right) \frac{\delta}{\lambda^{e}}\right] \\
& >\frac{\delta}{\lambda^{e}}\left[1-\frac{\lambda^{e}}{\delta} \frac{\delta}{\lambda^{e}}\right] \\
& =0 .
\end{aligned}
$$

Thus, $d \chi / d \gamma<0$ regardless of whether or not there is a binding minimum wage.

Average wage. Following Hornstein et al. (2011), the average wage in the model can be written as

$$
\begin{aligned}
E[w] & =\int_{\underline{w}}^{\bar{w}} w d G(w)=[w G(w)]_{\underline{w}}^{\bar{w}}-\int_{\underline{w}}^{\bar{w}} G(w) d w \\
& =\underline{w}+\int_{\underline{w}}^{\bar{w}}(1-G(w)) d w
\end{aligned}
$$

where, from the text, we have

$$
\begin{aligned}
& G(w)=\delta / \lambda^{e}\left(\frac{1-\sqrt{\frac{p-w}{p-\underline{w}}}}{\sqrt{\frac{p-w}{p-\underline{w}}}}\right) \\
& \bar{w}=p-(p-\underline{w})\left(\frac{1}{1+\lambda^{e} / \delta}\right)^{2} .
\end{aligned}
$$

As before, it is sufficient to establish that $d E[w] / d \gamma<0$ for the two relevant cases: a non-binding minimum wage and a binding minimum wage. We first consider the case of a non-binding minimum wage. In this case, note that we can use (18) in (5) to write the reservation wage as

$$
R=b+\frac{\lambda^{n}-\lambda^{e}}{\delta+\lambda^{e}} \int_{R}^{\bar{w}}(1-G(w)) d w
$$

Substituting (B.16) into (B.19), we obtain

$$
R=\rho E[w]+\frac{\lambda^{n}-\lambda^{e}}{\delta+\lambda^{e}}[E[w]-R]
$$


which can be rearranged to express the average wage as a function of the reservation wage

$$
\begin{aligned}
E[w] & =\frac{R\left(\delta+\lambda^{n}\right)-b\left(\delta+\lambda^{e}\right)}{\lambda^{n}-\lambda^{e}} \\
& =\frac{\lambda^{e}\left(\delta+\lambda^{n}\right) p+\delta\left(\delta+\lambda^{e}\right) b}{\left(\delta+\lambda^{e}\right)^{2}+\lambda^{e}\left(\lambda^{n}-\lambda^{e}\right)}
\end{aligned}
$$

where the second line uses the expression for the reservation wage in (21). Differentiating with respect to $\gamma$, taking account of (i) the dependence of $\lambda^{n}=\lambda^{n}(Z)$ on $\gamma$ via $Z$, and (ii) the dependence of $\lambda^{e}=\lambda^{e}(Z ; \gamma)$ on $\gamma$, both indirectly via $Z$ (the first argument) and directly (the second argument), we can write

$$
\begin{aligned}
\frac{d E[w]}{d \gamma} & \propto \delta\left(\delta+\lambda^{n}\right) \frac{d \lambda^{e}}{d \gamma}+\lambda^{e}\left(\delta+\lambda^{e}\right) \frac{d \lambda^{n}}{d \gamma} \\
& =\delta\left(\delta+\lambda^{n}\right)\left[\lambda_{\gamma}^{e}+\lambda_{Z}^{e} \frac{d Z}{d \gamma}\right]+\lambda^{e}\left(\delta+\lambda^{e}\right)\left[\lambda_{Z}^{n} \frac{d Z}{d \gamma}\right]
\end{aligned}
$$

where, as before, $\lambda_{\gamma}^{e}$ is the partial derivative of $\lambda^{e}$ with respect to its second argument. Note that, as currently written, the sign of this expression is ambiguous because $d \lambda^{e} / d \gamma<0$ but $d \lambda^{n} / d \gamma>0$. Noting that (B.1) implies that $d Z / d \gamma$ is linear in $\lambda_{\gamma}^{e}$, the preceding can be written as

$$
\frac{d E[w]}{d \gamma} \propto \lambda_{\gamma}^{e}\left[\delta\left(\delta+\lambda^{n}\right)\left[1+\lambda_{Z}^{e} a\right]+\lambda^{e}\left(\delta+\lambda^{e}\right) \lambda_{Z}^{n} a\right]
$$

where

$$
a \equiv \frac{d Z / d \gamma}{\lambda_{\gamma}^{e}}=\frac{r_{z}^{n} \Pi_{\lambda^{e}}}{c^{\prime \prime}(Z)-r_{z}^{n}\left(\Pi_{\lambda^{e}} \lambda_{Z}^{e}+\Pi_{\lambda^{n}} \lambda_{Z}^{n}\right)-\Pi_{z Z}^{n}}<0
$$

and the inequality follows from $d Z / d \gamma>0$ and $\lambda_{\gamma}^{e}<0$. To show that $d E[w] / d \gamma<0$, it is sufficient to show that the bracketed term in (B.25) is positive, i.e.

$$
\delta\left(\delta+\lambda^{n}\right)\left[1+\lambda_{Z}^{e} a\right]+\lambda^{e}\left(\delta+\lambda^{e}\right) \lambda_{Z}^{n} a>0 .
$$

Using the definition of $a$ and simplifying, we can rewrite the inequality as

$$
c^{\prime \prime}(Z)>r_{z}^{n} \lambda_{Z}^{n}\left[-\Pi_{\lambda^{e}} \frac{\lambda^{e}\left(\delta+\lambda^{e}\right)}{\delta\left(\delta+\lambda^{n}\right)}+\Pi_{\lambda^{n}}\right]+\Pi r_{z Z}^{n}
$$

Using (A.18) and (A.19), the bracketed term in (B.28) is zero, while (A.17) implies that the final term can be written as $r_{z Z}^{n}=-\frac{r_{z}^{n}}{Z}\left(1-(1-e) \epsilon_{Z}^{\lambda^{n}}\right)$. Noting also that the first-order condition for $z$ in (A.14) implies that $c^{\prime}(Z)=\Pi r_{Z}^{n}$, the inequality reduces to

$$
c^{\prime \prime}(Z) Z+c^{\prime}(Z)\left(1-(1-e) \epsilon_{Z}^{\lambda^{n}}\right)>0
$$

where the sign follows from concavity of $\lambda^{n}$ and convexity of the recruitment cost function. Thus, it must be that $d E[w] / d \gamma<0$. 
Next we consider the case of a binding minimum wage, $\underline{w}=w_{\min }$. First observe that (B.16) implies that $E[w]$ is increasing in $\bar{w}$ and decreasing in $G(w)$. Because neither $\bar{w}$ nor $G(w)$ depends on $\lambda^{n}$, it follows that $E[w]$ does not depend on $\lambda^{n}$, so it is sufficient to consider the effect of $\gamma$ through $\lambda^{e}$. From (22), we see that $\bar{w}$ is increasing in $\lambda^{e}$. From (18), we see that $G(w)$ is decreasing in $\lambda^{e}$. Together, these observations imply that $E[w]$ is increasing in $\lambda^{e}$ and thus decreasing in $\gamma$. That is, $d E[w] / d \gamma<0$.

Wage dispersion. In the case of a binding minimum wage, $M m=E[w] / w_{\min }$. The minimum wage $w_{\min }$ is a parameter of the environment (and is thus unaffected by NCAs), and we have already proven that NCAs reduce average wages, $E[w]$, so it follows that NCAs must reduce wage dispersion.

In the case of a non-binding minimum wage, we can rearrange (B.20) to obtain the expression for the mean-min wage ratio in the text:

$$
M m=\frac{1+\frac{\lambda^{n}-\lambda^{e}}{\delta+\lambda^{e}}}{\rho+\frac{\lambda^{n}-\lambda^{e}}{\delta+\lambda^{e}}}
$$

Differentiating with respect to $\gamma$ yields

$$
\frac{d M m}{d \gamma}=\frac{\frac{\rho-1}{\left(\delta+\lambda^{e}\right)^{2}}\left[\left(\delta+\lambda^{e}\right) \frac{d \lambda^{n}}{d \gamma}-\left(\delta+\lambda^{n}\right) \frac{d \lambda^{e}}{d \gamma}\right]-\left(\frac{\delta+\lambda^{n}}{\delta+\lambda^{e}}\right) \frac{d \rho}{d \gamma}}{\left(\rho+\frac{\lambda^{n}-\lambda^{e}}{\delta+\lambda^{e}}\right)^{2}}
$$

Note that $d \lambda^{n} / d \gamma>0$ (because NCAs increase recruitment by Proposition 1), $d \lambda^{e} / d \gamma<0$ (Proposition 2) and $d \rho / d \gamma>0$ (because $\rho \equiv b / E[w]$ and average wages are decreasing in NCAs as we have seen above). To complete the proof, consider two cases: $\rho \leq 1$ and $\rho>1$. If $\rho \leq 1$, inspection of (B.31) together with the signs of the derivatives of $\lambda^{n}, \lambda^{e}$ and $\rho$ with respect to $\gamma$ reveal immediately that $d M m / d \gamma<0$. On the other hand, if $\rho>1$, then $E[w]<b$, which implies that $R<b$ because the average wage must exceed the lowest wage in the economy ( $R$, absent a minimum wage), which in turn implies that $\lambda^{e}>\lambda^{n}$ (see equation (5)). However, if we differentiate (21) with respect to $\gamma$, we obtain

$$
\frac{d R}{d \gamma}=\frac{(p-b) \lambda^{e}\left(\delta+\lambda^{e}\right)^{2}}{\left(\left(\delta+\lambda^{e}\right)^{2}+\lambda^{e}\left(\lambda^{n}-\lambda^{e}\right)\right)^{2}} \frac{d \lambda^{n}}{d \gamma}-\frac{(p-b)\left(\delta+\lambda^{e}\right)\left(2 \delta \lambda^{e}-\delta \lambda^{n}+\lambda^{e} \lambda^{n}\right)}{\left(\left(\delta+\lambda^{e}\right)^{2}+\lambda^{e}\left(\lambda^{n}-\lambda^{e}\right)\right)^{2}} \frac{d \lambda^{e}}{d \gamma}
$$

Recalling that $d \lambda^{n} / d \gamma>0$ and $d \lambda^{e} / d \gamma<0$, this expression is only positive if the numerator of the second term is positive, which is necessarily the case since we know that $\lambda^{e}>\lambda^{n}$. Thus, for $\rho>1$, it must be that NCAs increase the reservation wage $R$. But because NCAs also reduce average wages $E[w]$, they must reduce the mean-min ratio, $M m=E[w] / R$.

\section{B.3 Minimum wage and recruitment}

Proposition B1 (Minimum wage and recruitment). Recruitment is weakly decreasing in the level of the minimum wage. 
Proof. Because $Z$ is continuous in $w_{\min }$, to establish that recruitment is weakly decreasing in NCA enforceability, it is sufficient to establish that $d Z / d w_{\min } \leq 0$ for the two relevant cases: a non-binding minimum wage and a binding minimum wage.

If the minimum wage is non-binding, then we have $\underline{w}=R$, so it must be the case that $d Z / d w_{\min }=0$. If the minimum wage is binding, then we can differentiate (A.14), taking account of the implicit dependence of $Z$ on $w_{\min }$, and solve for $d Z / d w_{\min }$, which gives

$$
\frac{d Z}{d w_{\min }}=\frac{r_{z}^{n} \Pi_{w_{\min }}}{c^{\prime \prime}(Z)-r_{z}^{n}\left(\Pi_{\lambda^{e}} \lambda_{Z}^{e}+\Pi_{\lambda^{n}} \lambda_{Z}^{n}\right)-\Pi r_{z Z}^{n}}<0
$$

where the sign follows from (i) convexity of $c(\cdot)$ which implies $c^{\prime \prime}(Z)>0$, (ii) (A.15)-(A.19) from which we know $\Pi>0, r_{z}^{n}>0, r_{z Z}^{n}<0, \Pi_{\lambda^{e}}<0$ and $\Pi_{\lambda^{n}} \leq 0$, (iii) the maintained assumptions that $\lambda_{Z}^{e}>0$ and $\lambda_{Z}^{n}>0$, and (iv) equation (A.15) from which we see that $\Pi_{w_{\min }}=\frac{-1}{\delta+\lambda^{e}(Z ; \gamma)}<0$. 


\section{Efficiency}

\section{C.1 Propositions from text}

\section{C.1.1 Proposition 5 (Efficiency)}

Proof. Equating the right-hand side of (31) (the planner's first-order condition) with the right-hand side of (17) (the first-order condition for a firm choosing $w \in[\underline{w}, \bar{w}]$ ), we obtain

$$
e^{\prime}(Z)(p-b)=\frac{p-w}{\delta+\lambda^{e}(Z ; \gamma)(1-H(w))}\left[\frac{(1-e) \lambda^{n}(Z)+e \lambda^{e}(Z ; \gamma) G(w)}{Z}\right] .
$$

for any $w \in[\underline{w}, \bar{w}]$. Multiplying both sides by $Z$, dividing both sides by $e$, and noting that $\epsilon_{Z}^{e}(Z) \equiv e^{\prime}(Z) Z / e(Z)$, this is

$$
\epsilon_{Z}^{e}(Z)(p-b)=\frac{p-w}{\delta+\lambda^{e}(Z ; \gamma)(1-H(w))}\left[\frac{1-e}{e} \lambda^{n}(Z)+\lambda^{e}(Z ; \gamma) G(w)\right] .
$$

Dividing both sides by $p-b$, multiplying and dividing the right-hand side by $\delta$, and noting that $\frac{1-e}{e} \lambda^{n}(Z) / \delta=1$, we can rewrite the previous equation as

$$
\epsilon_{Z}^{e}(Z)=\frac{\frac{p-w}{\delta+\lambda^{e}(Z ; \gamma)(1-H(w))}}{\frac{p-b}{\delta}}\left[1+\frac{\lambda^{e}(Z ; \gamma) G(w)}{\delta}\right]
$$

Finally, using $\Pi(w ; Z) \equiv \frac{p-w}{\delta+\lambda^{e}(Z ; \gamma)(1-H(w))}, \Pi^{S P} \equiv \frac{p-b}{\delta}$, and using the definitions of $r^{e}(w, Z)$ and $r^{n}(Z)$ in Section 3.3, which imply that $\phi(w ; Z) \equiv r^{e}(w, Z) / r^{n}(Z)=\frac{\lambda^{e}(Z ; \gamma) G(w)}{\delta}$, we can rearrange to obtain

$$
\frac{1+\phi(w ; Z)}{\epsilon_{Z}^{e}(Z)}=\frac{\Pi^{S P}}{\Pi(w ; Z)} .
$$

This is the general efficiency condition for any $w \in[\underline{w}, \bar{w}]$. To recover the condition in (32), evaluate (C.4) at $w=\underline{w}$ and note that $\phi(\underline{w} ; Z)=0$, which implies

$$
\frac{1}{\epsilon_{Z}^{e}(Z)}=\frac{\Pi^{S P}}{\Pi(\underline{w} ; Z)}
$$

Profit equalization and the fact that all firms choose the same $z=Z$ implies that these conditions are equivalent in terms of their implications for efficiency.

\section{C.1.2 Proposition 6 (Necessity and sufficiency of two instruments)}

Proof. We first prove that two instruments are necessary to guarantee that efficiency can be achieved, and then prove that two instruments are always sufficient to achieve efficiency.

Necessity: We first prove that neither NCAs nor a minimum wage alone can guarantee efficiency. In both cases, it is sufficient to find examples of economies in which, with a single instrument, 
policy makers cannot implement the efficient allocation. First, consider the case in which policy makers can only set NCA policy $(\gamma)$ and there is no minimum wage. Consider an economy in which (i) the offer rate for non-employed workers is strictly concave in $Z$ (i.e. $\epsilon_{Z}^{\lambda^{n}}<1$ ), and (ii) absent NCAs, the offer rate functions are symmetric for employed and non-employed workers (i.e. $\left.\lambda^{n}(Z)=\lambda^{e}(Z ; 0)\right)$. Symmetry of the offer rate functions for $\gamma=0$ implies that $\underline{w}=R=b$. Recalling that $\Pi(\underline{w} ; Z) \equiv \frac{p-\underline{w}}{\delta+\lambda^{e}(Z ; \gamma)}$ and $\Pi^{\mathrm{SP}} \equiv \frac{p-b}{\delta}$, the preceding implies that for $\gamma=0$,

$$
\Pi(\underline{w} ; Z) / \Pi^{\mathrm{SP}}=\frac{\delta}{\delta+\lambda^{e}(Z ; 0)}=\frac{\delta}{\delta+\lambda^{n}(Z)}=1-e>\epsilon_{Z}^{\lambda^{n}}(1-e)
$$

where the second equality uses equality of the offer functions when $\gamma=0$, the third equality uses the definition of $e$ in (9), and the inequality follows from strict concavity of $\lambda^{n}(Z)$. Thus, from the efficiency condition in (32), there must be excess recruitment if $\gamma=0$. But because recruitment is increasing in $\gamma$ (Proposition 1) and $\gamma$ must be weakly positive (Assumption A2), NCA policy can only increase recruitment. Thus, it is impossible to eliminate excess recruitment in order to achieve efficiency.

Next, consider the case in which policy makers can only set minimum wage policy $\left(w_{\min }\right)$ and in which there are no NCAs. Suppose the offer rate function for non-employed workers is linear $\left(\epsilon_{Z}^{\lambda^{n}}=1\right)$. In this case, if the minimum wage is non-binding, then using the expression for the reservation wage in (21), the efficiency condition in (32) implies that there is insufficient recruitment if and only if $\lambda^{e}(Z ; \gamma)>\lambda^{n}(Z) .{ }^{41}$ Suppose frictions in the labor market are such that this condition is satisfied (this will be the case, for example, if $\lambda^{e}(Z ; 0)=\mu^{e} Z$ and $\lambda^{n}(Z)=\mu^{n} Z$ with $\mu^{e}>\mu^{n}$ ). Then, there will trivially be insufficient recruitment for any non-binding minimum wage (because the minimum wage has no effect on the economy when it is non-binding), whereas any binding minimum wage will necessarily reduce recruitment from its level associated with a non-binding minimum wage by Proposition B1. Thus, it is impossible to stimulate recruitment in order to achieve efficiency.

Sufficiency: We next establish that, with both instruments, efficiency can always be achieved. We first consider the case of an economy with insufficient recruitment and then consider an economy with excessive recruitment. In both cases, we show that it is possible to construct a policy configuration $\left(\gamma^{*}, w_{\min }^{*}\right)$ that implements the efficient allocation.

Consider first an economy with $\left(\gamma, w_{\min }\right)$ such that there is insufficient recruitment. If elimination of the minimum wage results in excessive recruitment, then continuity of $Z$ in $w_{\text {min }}$ implies that there must be some $w_{\min }^{*}<w_{\min } \operatorname{such}$ that $\left(\gamma, w_{\min }^{*}\right)$ implements the efficient allocation. If there is still insufficient recruitment after eliminating the minimum wage, then (i) it must be that $\gamma<1$, implying that there is scope for increasing NCAs, and (ii) there must be some $\gamma^{*} \in(\gamma, 1)$ such that $\left(\gamma^{*},-\infty\right)$ implements the social optimum. To understand why these two claims are true, consider the limit economy with no minimum wage and with fully restrictive NCAs $(\gamma \rightarrow 1)$. In this economy, $\lambda^{e}=0$ (by Assumption A3), which implies that $\underline{w}=R=b$ and thus $\Pi(\underline{w} ; Z)=\Pi^{\mathrm{SP}}$, so we have

$$
\Pi(\underline{w} ; Z) / \Pi^{\mathrm{SP}}=1 \geq \epsilon^{\lambda_{Z}^{n}}>(1-e) \epsilon_{Z}^{\lambda^{n}}=\epsilon_{Z}^{e}
$$

\footnotetext{
${ }^{41}$ This can be verified more easily using (C.11) with $\epsilon_{Z}^{\lambda^{n}}=1, \underline{w}=R$ and $e=\lambda^{n} /\left(\delta+\lambda^{n}\right)$.
} 
where the first inequality follows from weak concavity of $\lambda^{n}$, the second inequality follows from the fact that employment is always positive with positive recruitment, and the final equality is just the expression for $\epsilon_{Z}^{e}$ that we derived in the text. Thus, by Proposition 5, the $\gamma \rightarrow 1$ limit economy with no minimum wage must have excessive recruitment. This, in turn, implies that any economy with insufficient recruitment and a non-binding minimum wage must have $\gamma<1$ (as claimed), and furthermore, due to Proposition 1 and continuity of $Z$ in $\gamma$, there must exist some $\gamma^{*} \in(\gamma, 1)$ such that $\left(\gamma^{*},-\infty\right)$ achieves efficiency (as claimed).

Consider next an economy with $\left(\gamma, w_{\min }\right)$ such that there is excessive recruitment. The existence of excessive recruitment implies that (i) it must be that $w_{\min }<p$, which in turn implies that there is scope for increasing the minimum wage, and (ii) there must exist some $w_{\min }^{*} \in\left(w_{\min }, p\right)$ such that $\left(\gamma, w_{\min }^{*}\right)$ achieves efficiency. To understand why these claims are true, consider the $w_{\min } \rightarrow p$ limit economy. In this economy, we immediately see that $\Pi\left(w_{\min } ; Z\right) \equiv \frac{p-w_{\min }}{\delta+\lambda^{e}}=\frac{p-p}{\delta+\lambda^{e}}=0$, so we have

$$
\Pi(\underline{w} ; Z) / \Pi^{\mathrm{SP}}=0<(1-e) \epsilon_{Z}^{\lambda^{n}}=\epsilon_{Z}^{e}
$$

where the inequality follows from the fact that $\lambda^{n}$ is strictly increasing in $Z$, implying that $\epsilon_{Z}^{\lambda^{n}}>0$. Thus, by Proposition 5, the $w_{\min } \rightarrow p$ limit economy must have insufficient recruitment. This, in turn, implies that any economy with excessive recruitment must have $w_{\min }<p$ (as claimed), and furthermore, by Proposition B1 and continuity of $Z$ in $w_{\min }$, there must exist some $w_{\min }^{*} \in\left(w_{\min }, p\right)$ that achieves efficiency.

\section{C.1.3 Proposition 7 (Sufficient conditions for inefficiency of NCAs)}

Proof. The condition for efficiency in (32) (evaluated at $w=\underline{w}$ ) implies that there is excess recruitment if and only if: ${ }^{42}$

$$
\epsilon_{z}^{e}<\frac{\Pi(\underline{w}(Z) ; Z)}{\Pi^{\mathrm{SP}}}
$$

Using the definitions of $\Pi(\underline{w}(Z) ; Z)$ and $\Pi^{\mathrm{SP}}$, and the fact that $\epsilon_{Z}^{e}=(1-e) \epsilon_{Z}^{\lambda^{n}}$, the condition for excess recruitment can be written as

$$
(1-e) \epsilon_{Z}^{\lambda^{n}}<\frac{\left(\frac{p-\underline{w}}{\delta+\lambda^{e}}\right)}{\left(\frac{p-b}{\delta}\right)}=\frac{\left(\frac{p-R}{\delta+\lambda^{e}}\right)}{\left(\frac{p-b}{\delta}\right)}\left(\frac{p-\underline{w}}{p-R}\right) .
$$

Using the expression for the reservation wage in (21) to eliminate $R$ from $\left(\frac{p-R}{\delta+\lambda^{e}}\right) /\left(\frac{p-b}{\delta}\right)$ (but not from $\frac{p-\underline{w}}{p-R}$ ), using the definition of $e$ in (9), and rearranging, this condition can be written as

$$
1-\frac{\epsilon_{Z}^{\lambda^{n}}}{\left(\frac{p-\underline{w}}{p-R}\right)}(1-e)>\frac{\lambda^{e}}{\delta}\left[\frac{\epsilon_{Z}^{\lambda^{n}}}{\left(\frac{p-\underline{w}}{p-R}\right)}(2-e)-1\right]
$$

\footnotetext{
${ }^{42}$ For ease of notation, we once again suppress dependence of endogenous variables on $Z$ and $\gamma$.
} 
Notice that if the right-hand side of (C.11) is negative, then the left-hand side must be positive:

$$
\begin{aligned}
& \frac{\epsilon_{Z}^{\lambda^{n}}}{\left(\frac{p-\underline{w}}{p-R}\right)}(2-e)-1<0 \\
\Longrightarrow & 1-\frac{\epsilon_{Z}^{\lambda^{n}}}{\left(\frac{p-\underline{w}}{p-R}\right)}(1-e)>\frac{\epsilon_{Z}^{\lambda^{n}}}{\left(\frac{p-\underline{w}}{p-R}\right)}>0 .
\end{aligned}
$$

Thus, (C.12) is a sufficient condition for the inequality in (C.11) to hold. Rearranging (C.12), we can express the condition as a threshold for the employment rate, yielding the expression in (33):

$$
e>2-\frac{1}{\epsilon_{Z}^{\lambda^{n}}}\left(\frac{p-\underline{w}}{p-R}\right)
$$

To obtain (34), note that if the minimum wage does not bind, then $\underline{w}=R$, so (C.14) becomes

$$
e>2-\frac{1}{\epsilon_{Z}^{\lambda^{n}}}
$$

To obtain (35), note that if the minimum wage is binding (i.e. $\underline{w}=w_{\min }$ ), then for $R>0$ the condition in (C.14) is implied by ${ }^{43}$

$$
\begin{aligned}
e & >2-\frac{1}{\epsilon_{Z}^{\lambda^{n}}}\left(\frac{p-w_{\min }}{p}\right) \\
& =2-\frac{1}{\epsilon_{Z}^{\lambda^{n}}}\left(1-\frac{w_{\min }}{p}\right) \\
& =2-\frac{1}{\epsilon_{Z}^{\lambda^{n}}}\left(1-\frac{E[w] / p}{E[w] / w_{\min }}\right) \\
& =2-\frac{1}{\epsilon_{Z}^{\lambda^{n}}}\left(1-\frac{\eta^{L}}{M m}\right)
\end{aligned}
$$

where $\eta^{L} \equiv E[w] / p$ is labor's share and $M m \equiv E[w] / w_{\min }$ is the mean-min wage ratio.

\footnotetext{
${ }^{43}$ Although it is possible that $R<0$, this does not occur in any plausible region of the parameter space.
} 


\section{Calibration}

This appendix describes the calibration of the model. ${ }^{44}$ First, as described in Table 1, we directly calibrate several parameters: $\lambda^{n}=0.17$ is chosen to match the monthly job-finding probability of non-employed individuals who report wanting a job in the CPS from Hall and Schulhofer-Wohl $(2018){ }^{45} b=0.73$ is chosen to match the midpoint of the range of estimates of the opportunity cost of employment for workers without a high school diploma in Chodorow-Reich and Karabarbounis (2016); $\epsilon_{Z}^{\lambda^{n}}=0.44$ is chosen to match the estimated elasticity of matches from the non-employed population that reports wanting a job (consistent with our calibration of $\lambda^{n}$ above) with respect to vacancies from Hall and Schulhofer-Wohl (2018), ${ }^{46} \epsilon_{Z}^{\lambda^{e}}=0.26$ is chosen to match the estimated elasticity of matches from the already-employed population with respect to vacancies, again from Hall and Schulhofer-Wohl (2018); and $\epsilon_{Z}^{c}=2$ is chosen such that the recruitment cost function is quadratic.

The remaining parameters are calibrated indirectly to match various moments in the data. ${ }^{47}$ First, we choose the job destruction rate, $\delta$, to match the employment-to-population ratio for the prime-age (25-54) civilian population without a high school diploma in Oregon in 2006, $\hat{e}=0.65$, using (9): ${ }^{48}$

$$
\delta=\lambda^{n}\left(\frac{1-\hat{e}}{\hat{e}}\right)
$$

Next, following Hornstein et al. (2011), we choose the offer rate for employed workers, $\lambda^{e}$, to match the average rate of job-to-job transitions for workers without a high school degree in Fallick and Fleischman (2001), $\hat{\chi}=0.035$. Specifically, $\lambda^{e}$ is determined by the unique implicit solution to (25):

$$
\hat{\chi}=\delta\left[\frac{\delta+\lambda^{e}}{\lambda^{e}} \ln \left(\frac{\delta+\lambda^{e}}{\delta}\right)-1\right] .
$$

We choose the minimum wage, $w_{\min }$, to match the ratio of the minimum wage to the median wage among full-time prime-age (25-54) civilian workers without a high school diploma in Oregon in 2006 (consistent with our computation of the employment-to-population ratio above), $\hat{r}^{\mathrm{min} / \mathrm{med}} \equiv$ $w_{\text {min }} / w_{\text {median }}=0.75$. Specifically, noting that the median wage solves $G(w)=0.5$ when the minimum wage is binding, if the minimum wage is binding (which we check below) we can write

$$
w_{\min }^{\mathrm{B}}=\frac{\hat{r}^{\mathrm{min} / \operatorname{med}} p\left(1-\left(1+0.5 \lambda^{e} / \delta\right)^{-2}\right)}{1-\hat{r}^{\min / \operatorname{med}}\left(1+0.5 \lambda^{e} / \delta\right)^{-2}} .
$$

Furthermore, the parameter values that we have chosen up to this point allow us to pin down the reservation wage, $R$, via (21). If $R$ is less than $w_{\min }^{\mathrm{B}}$, then the minimum wage is binding and we

\footnotetext{
${ }^{44}$ For ease of notation, we suppress dependence of endogenous variables on $Z$ and $\gamma$ throughout.

${ }^{45}$ Average job-finding rates generally do not differ a great deal between individuals with different levels of education.

${ }^{46}$ This value is similar to the elasticity of matches from non-employment with respect to vacancies in Veracierto (2011) of 0.38 .

${ }^{47}$ Where applicable, we denote empirical analogs to model objects with hats.

${ }^{48}$ This value is based on the authors' calculations from CPS data.
} 
proceed with calibration. If $R$ is greater than $w_{\text {min }}^{\mathrm{B}}$, then we use the identified value of $R$ to compute the model-implied median wage via

$$
w_{\text {median }}=p-(p-R)\left(1+0.5 \lambda^{e} / \delta\right)^{-2}
$$

from which we can recover the value of the non-binding minimum wage via

$$
w_{\min }^{\mathrm{NB}}=\hat{r}^{\mathrm{min} / \operatorname{med}} w_{\text {median }} .
$$

Thus, if $R<w_{\min }^{\mathrm{B}}$, then $\underline{w}=w_{\min }=w_{\min }^{\mathrm{B}}$, and if $R>w_{\min }^{\mathrm{B}}$, then $\underline{w}=R$ and $w_{\min }=w_{\min }^{\mathrm{NB}}$. Note that it will be important that we have a value of the minimum wage, even if it is non-binding, for our quantitative analysis of the interaction between NCAs and the minimum wage. Next, we choose the NCA parameter, $\gamma$, to match the midpoint of the observed effect of Oregon's 2008 ban on NCAs among low-wage workers on the average rate of job-to-job transitions documented by Lipsitz and Starr (2021), $\hat{\epsilon}_{\gamma}^{\chi} \equiv \frac{\chi_{\mathrm{Ban}}-\chi_{\mathrm{NCA}}}{\chi_{\mathrm{NCA}}}=0.15$. This is done by noting that the preceding implies that $\chi_{\mathrm{Ban}}=\left(1+\hat{\epsilon}_{\gamma}^{\chi}\right) \hat{\chi}$ (recall that $\hat{\chi}$ is the average job-to-job rate computed by Fallick and Fleischman (2001) based on data from before 2008), so if we define $\lambda_{\mathrm{Ban}}^{e} \equiv\left(1-\gamma_{\mathrm{Ban}}\right) \mu^{e} Z^{\epsilon_{Z}^{\lambda^{e}}}=(1-0) \mu^{e} Z^{\epsilon_{Z}^{e}}=\mu^{e} Z^{\epsilon_{Z}^{\lambda^{e}}}$ (that is, the offer rate absent NCAs), we can compute $\lambda_{\mathrm{Ban}}^{e}$ as the solution to the equation relating offer rates for employed workers to the average job-to-job transition rate in (25) for the post-ban period:

$$
\chi_{\mathrm{Ban}}=\delta\left[\frac{\delta+\lambda_{\mathrm{Ban}}^{e}}{\lambda_{\mathrm{Ban}}^{e}} \ln \left(\frac{\delta+\lambda_{\mathrm{Ban}}^{e}}{\delta}\right)-1\right]
$$

which allows us to solve for $\gamma$ via

$$
\gamma=1-\frac{\lambda^{e}}{\lambda_{\text {Ban }}^{e}}
$$

From here, it remains to solve for $c_{0}, \mu^{n}$ and $\mu^{e}$. To do so, we use data on hiring costs as a percentage of monthly pay from Barron et al. (1997), as reported in the second row of Table 2 of Manning (2011). Specifically, Barron et al. (1997) find that $\hat{\psi} \equiv E\left[\frac{c^{\prime}(Z)}{w}\right]$ is between 0.05 and 0.14 , so we use the midpoint: $\hat{\psi}=\frac{0.05+0.14}{2}$. Using this and writing $\hat{\psi}=\int_{\underline{w}}^{\bar{w}} \frac{c^{\prime}(Z)}{w} h(w) d w=c^{\prime}(Z) \int_{\underline{w}}^{\bar{w}} \frac{1}{w} h(w) d w$, which implies that $c^{\prime}(Z)=\hat{\psi}\left[\int_{\underline{w}}^{\bar{w}} \frac{1}{w} h(w) d w\right]^{-1}$, we can rearrange the first-order condition in $(23)$ to solve for $Z$ :

$$
Z=\frac{\int_{\underline{w}}^{\bar{w}} \frac{1}{w} h(w) d w \frac{p-\underline{w}}{\delta+\lambda^{e}} \delta \lambda^{n}}{\delta+\lambda^{n}} .
$$

From here, we can use the recruitment cost functional form (which implies that $c^{\prime}(Z)=c_{0} Z_{Z}^{\epsilon_{Z}^{c}-1}$ ) together with the first-order condition in (23) to solve for $c_{0}$,

$$
c_{0}=\frac{\frac{p-\underline{w}}{\delta+\lambda^{e}} \frac{\delta \lambda^{n}}{\delta+\lambda^{n}}}{Z^{\epsilon_{Z}^{c}}}
$$


Finally, we can use the offer rate functions to solve for the offer rate scale parameters,

$$
\begin{aligned}
\mu^{n} & =\frac{\lambda^{n}}{Z_{Z}^{\epsilon^{n}}} \\
\mu^{e} & =\frac{\lambda^{e}}{(1-\gamma) Z_{Z}^{\epsilon^{\lambda^{e}}}}
\end{aligned}
$$




\section{E Free Entry}

The model presented in the body of the text studies the implications of NCAs for firms' recruitment decisions, taking as given the number of firms in the economy. In this appendix, we show that all of the qualitative comparative statics and efficiency results continue to hold in an otherwise identical model in which, rather than having a fixed number of firms making endogenous recruitment decisions, there is an endogenous number of firms determined by a free-entry condition whose recruitment intensity is fixed.

\section{E.1 Model}

Consider an identical model to the one described in the body of the text, but in which (i) there is no recruitment decision, (ii) the economy is populated by an endogenous measure $M_{f}$ of firms such that $\lambda^{e}=\lambda^{e}\left(M_{f} ; \gamma\right)$ and $\lambda^{n}=\lambda^{n}\left(M_{f}\right)$ where both functions satisfy the properties described in Section 3.1.1 (now with respect to $M_{f}$ rather than $Z$ ), and (iii) entry requires payment of fixed cost $C_{f}>0 .{ }^{49}$ Notice that in this model, the measure of firms $M_{f}$ plays an identical role to aggregate recruitment intensity $Z$ in the model in the body of the text in terms of its influence on the offer rates $\lambda^{n}$ and $\lambda^{e} .{ }^{50}$

The alternative model requires only minimal modification to the model in the text. Specifically, the inflow of recruits to a firm choosing wage $w$ in (10) is now given by

$$
r(w)=r^{e}\left(w, M_{f}\right)+r^{n}\left(M_{f}\right)=\frac{\lambda^{e}\left(M_{f} ; \gamma\right) e\left(M_{f}\right) G(w)+\lambda^{n}\left(M_{f}\right)\left(1-e\left(M_{f}\right)\right)}{M_{f}}
$$

implying that the labor supply to a firm offering wage $w$ is given by

$$
l(w) \equiv \frac{\lambda^{e}\left(M_{f} ; \gamma\right) e\left(M_{f}\right) G(w)+\lambda^{n}\left(M_{f}\right)\left(1-e\left(M_{f}\right)\right)}{M_{f}\left[\delta+\lambda^{e}\left(M_{f} ; \gamma\right)(1-H(w))\right]}
$$

yielding profits (gross of $C_{f}$ ) of

$$
\pi\left(w, M_{f}\right)=(p-w) \frac{\lambda^{e}\left(M_{f} ; \gamma\right) e\left(M_{f}\right) G(w)+\lambda^{n}\left(M_{f}\right)\left(1-e\left(M_{f}\right)\right)}{M_{f}\left[\delta+\lambda^{e}\left(M_{f} ; \gamma\right)(1-H(w))\right]} .
$$

\footnotetext{
${ }^{49}$ For ease of exposition, the model presented in the body of the text implicitly assumes a unit measure of firms: $M_{f}=1$. This assumption has no effect on our qualitative analysis. Furthermore, it only affects our quantitative analysis by rescaling our recruitment cost scale parameter $c_{0}$. Because our calibration procedure targets hiring costs as a fraction of monthly pay, this rescaling has no effect on our quantitative results.

${ }^{50}$ See Manning (2003) for discussion of a similar model with symmetric offer rates.
} 


\section{E.2 Decentralized equilibrium}

Free entry implies that firms will enter until profits are equalized with the cost of entry. Evaluating (E.3) at $w=\underline{w}$, the free-entry condition that implicitly pins down $M_{f}$ is:

$$
C_{f}=\frac{p-\underline{w}}{M_{f}\left(\delta+\lambda^{e}\left(M_{f} ; \gamma\right)\right)}\left(1-e\left(M_{f}\right)\right) \lambda^{n}\left(M_{f}\right)
$$

Notice the similarity between equation (E.4) and equation (17) in the body of the text (repeated below for ease of comparison):

$$
c^{\prime}(Z)=\frac{p-\underline{w}}{Z\left(\delta+\lambda^{e}(Z ; \gamma)\right)}(1-e(Z)) \lambda^{n}(Z)
$$

Importantly, in the first equation $M_{f}$ is the endogenous variable, whereas in the second equation $Z$ is the endogenous variable.

To see that the two models yield identical qualitative comparative statics, it is sufficient to observe that (i) if $c(\cdot)$ is linear, then if we replace $M_{f}$ with $Z$, (E.4) is identical to (E.5) up to a scaling factor, and (ii) strict convexity of $c(\cdot)$ is only required for the existence of an interior solution to the firm's recruitment problem (i.e., the choice of $z$ given $Z$ ), which is not relevant in the free-entry model - otherwise, none of the qualitative results proved for the recruitment model in Appendices A or $\mathrm{B}$ depend on either strict convexity or the scale of $c(\cdot) .{ }^{51}$

\section{E.3 Welfare}

To see that the two models yield identical qualitative welfare implications, consider the planner's problem in the free-entry model:

$$
\Omega\left(M_{f}\right)=e\left(M_{f}\right) p+\left(1-e\left(M_{f}\right)\right) b-C_{f} M_{f}
$$

The planner now chooses $M_{f}$ instead of $Z$, yielding the optimality condition

$$
C_{f}=e^{\prime}\left(M_{f}\right)(p-b)
$$

Compare this with the planner's optimality condition in the recruitment model in (31):

$$
c^{\prime}(Z)=e^{\prime}(Z)(p-b) .
$$

Equating the right-hand side of (E.4) with the right-hand side of (E.7) yields the condition for efficiency in the free-entry model,

$$
\frac{p-\underline{w}}{M_{f}\left(\delta+\lambda^{e}\left(M_{f} ; \gamma\right)\right)}\left(1-e\left(M_{f}\right)\right) \lambda^{n}\left(M_{f}\right)=e^{\prime}\left(M_{f}\right)(p-b) .
$$

\footnotetext{
${ }^{51}$ Some of the results do depend on weak convexity of $c(\cdot)$, but obviously that does not rule out linearity.
} 
Likewise, equating the right-hand side of (E.5) with the right-hand side of (E.8) yields the condition for efficiency in the recruitment model,

$$
\frac{p-\underline{w}}{Z\left(\delta+\lambda^{e}(Z ; \gamma)\right)}(1-e(Z)) \lambda^{n}(Z)=e^{\prime}(Z)(p-b) .
$$

Thus, replacing $Z$ with $M_{f}$, we see that the two efficiency conditions are identical. 